\title{
A REGULARIZED SMOOTHING NEWTON METHOD FOR SYMMETRIC CONE COMPLEMENTARITY PROBLEMS*
}

\author{
LINGCHEN KONG ${ }^{\dagger}$, JIE SUN ${ }^{\ddagger}$, AND NAIHUA XIU ${ }^{\dagger}$
}

\begin{abstract}
This paper extends the regularized smoothing Newton method in vector complementarity problems to symmetric cone complementarity problems (SCCP), which includes the nonlinear complementarity problem, the second-order cone complementarity problem, and the semidefinite complementarity problem as special cases. In particular, we study strong semismoothness and Jacobian nonsingularity of the total natural residual function for SCCP. We also derive the uniform approximation property and the Jacobian consistency of the Chen-Mangasarian smoothing function of the natural residual. Based on these properties, global and quadratical convergence of the proposed algorithm is established.
\end{abstract}

Key words. symmetric cone complementarity problem, monotonicity, natural residual function, regularized smoothing method, quadratic convergence

AMS subject classifications. 65K05, 90C33

DOI. $10.1137 / 060676775$

1. Introduction. We are interested in the following symmetric cone complementarity problem (SCCP): Find vectors $x, y \in \mathcal{J}$ such that

$$
x \in K, \quad y=F(x) \in K,\langle x, y\rangle=0,
$$

where $\mathcal{J}$ is an $n$-dimensional real Euclidean space, $\mathcal{A}:=(\mathcal{J},\langle\cdot, \cdot\rangle, \circ)$ is a Euclidean Jordan algebra, $K$ is a symmetric cone in $\mathcal{A}$ (see section 2), and $F: \mathcal{J} \rightarrow \mathcal{J}$ is a continuously differentiable mapping. Problem (1.1) includes the semidefinite complementarity problem (SDCP), the second-order cone complementarity problem (SOCCP), and the nonlinear complementarity problem (NCP) as special cases. The SCCP has wide applications; in particular, it may arise from the KKT system of a nonlinear optimization problem. The SCCP has been the focal point of some recent research; see, e.g., $[12,13,14,22,23,29,33,37]$.

We intend to design an algorithm for SCCPs, which is called the regularized smoothing Newton method. In the setting of NCP, various regularized smoothing methods have been tested, which, in addition to their simplicity of implementation, have the advantage of being able to solve some ill-posed problems. Recently, there are some papers studying the smoothing Newton methods with or without regularization for SOCCP and SDCP; see, e.g., [3, 4, 5, 6, 7, 11, 15, 16, 32, 35]. These papers either address the case of SOCCP or that of SDCP, but to our knowledge, there are no discussions on this type of algorithms under the general framework of SCCP.

In this paper, with the help of the Euclidean Jordan algebra, we analyze the strong semismoothness and Jacobian nonsingularity of a natural residual function

*Received by the editors December 5, 2006; accepted for publication (in revised form) May 19, 2008; published electronically October 22, 2008. The work was partly supported by the National Natural Science Foundation of China (10671010, 70640420143), the Singapore-MIT Alliance, and Grant RP314000-057-112 of National University of Singapore.

http://www.siam.org/journals/siopt/19-3/67677.html

${ }^{\dagger}$ Department of Applied Mathematics, Beijing Jiaotong University, Beijing 100044, P. R. China (konglchen@126.com, nhxiu@center.njtu.edu.cn).

$\ddagger$ Department of Decision Sciences and Singapore-MIT Alliance, National University of Singapore, Republic of Singapore (jsun@nus.edu.sg).

1028 
(the so-called total NR-function). We also show the level-boundedness of the natural merit function of the total NR-function for SCCP under monotonicity and strict feasibility assumptions. We then construct the Chen-Mangasarian smoothing function of the natural residual for SCCP. Our work provides a theoretical and computational framework for solving general nonlinear SCCP. In particular, we derive the uniform approximation property and the Jacobian consistency of this smoothing function. These properties form a basis for establishing quadratic convergence of Newton-type algorithms. Finally, we state a globally and quadratically convergent algorithm for solving monotone SCCP that was originated from a similar algorithm of Hayashi, Yamashita, and Fukushima [15] for SOCCP. Many analytic tools we used are taken from the recent work by Sun and Sun [30], in which the differential properties of the Löwner's operator and spectral functions are studied in the space of Euclidean Jordan algebras.

This paper is organized as follows. In section 2, we briefly describe Euclidean Jordan algebra and some of its properties used in our analysis. We also derive new results on the Jacobian and the Clarke generalized Jacobian of Löwner operators. In section 3, we introduce and characterize the total NR-function for SCCP. In section 4, we present the Chen-Mangasarian smoothing function in the context of SCCPs and discuss its properties. In section 5, we introduce the regularized smoothing Newton method for SCCP and discuss its convergence.

The following notations will be used throughout this paper. Let $\mathcal{X}$ and $\mathcal{Y}$ be two finite dimensional real Euclidean spaces. For a given set $S \subseteq \mathcal{X}$, int $S$ and $\operatorname{conv} S$ denote the interior and convex hull of $S$, respectively. Let $\operatorname{dist}(a, S)$ be $\min \{\|a-b\|$ : $b \in S\}$ for $a \in \mathcal{X}$, where $\|\cdot\|$ is the norm on $\mathcal{X}$ induced by the inner product $\langle\cdot, \cdot \cdot$. We write $x \succeq_{K} y$ (respectively, $x \succ_{K} y$ ) to mean $x-y \in K$ (respectively, $x-y \in \operatorname{int} K$ ) for vectors $x, y \in \mathcal{J}$. Also, we write $A \succeq B(A \succ B)$ to mean $A-B$ being positive semidefinite (positive definite) for operators $A$ and $B$ from $\mathcal{J}$ into itself. Let $I$ be the identity operator, i.e., $I x=x$ for all $x \in \mathcal{J}$. We say that the operator $A$ is invertible (or nonsingular) if the equation $A x=0$ has a unique solution $x=0$. For an operator $A, A^{T}$ denotes the adjoint operator of $A$.

\section{Preliminaries.}

2.1. Euclidean Jordan algebras. We give a brief introduction to Euclidean Jordan algebras. Details on Euclidean Jordan algebras can be found in Koecher's lecture notes [19] and the monograph by Faraut and Korányi [10].

A Euclidean Jordan algebra (EJA) is a triple $(\mathcal{J},\langle\cdot, \cdot\rangle, \circ) \triangleq \mathcal{A}$, where $(\mathcal{J},\langle\cdot, \cdot\rangle)$ is a real $n$-dimensional inner product space and $(x, y) \mapsto x \circ y: \mathcal{J} \times \mathcal{J} \rightarrow \mathcal{J}$ is a bilinear mapping which satisfies the following conditions:

(i) $x \circ y=y \circ x$ for all $x, y \in \mathcal{J}$,

(ii) $x \circ\left(x^{2} \circ y\right)=x^{2} \circ(x \circ y)$ for all $x, y \in \mathcal{J}$ where $x^{2}:=x \circ x$,

(iii) $\langle x \circ y, z\rangle=\langle x, y \circ z\rangle$ for all $x, y, z \in \mathcal{J}$.

We call $x \circ y$ the Jordan product of $x$ and $y$. In general, the Jordan product is not associative; i.e., $(x \circ y) \circ z \neq x \circ(y \circ z)$ for all $x, y, z \in \mathcal{J}$. In addition, we assume that there exists an element $e$ (called the identity element) such that $x \circ e=e \circ x=x$ for all $x \in \mathcal{J}$. The following are some basic facts about Euclidean Jordan algebras.

- Given a Euclidean Jordan algebra $\mathcal{A}$, define the set of squares as $K:=\left\{x^{2}\right.$ : $x \in \mathcal{J}$ \}. From Theorem III 2.1 in [10], $K$ is a symmetric cone in $\mathcal{A}$. In other words, $K$ is a self-dual closed convex cone, and for any two elements $x, y \in \operatorname{int} K$, there exists an invertible linear transformation $\Gamma: \mathcal{J} \rightarrow \mathcal{J}$ such that $\Gamma(K)=K$ and $\Gamma(x)=y$. 
- For $x \in \mathcal{J}$, let $m:=m(x)$ be the smallest positive integer such that the set $\left\{e, x, x^{2}, \ldots, x^{m}\right\}$ is linearly dependent. Then $m$ is said to be the degree of $x$, which is denoted by $\operatorname{deg}(x)$.

- The rank of $\mathcal{A}$ denoted by $\operatorname{rk}(\mathcal{A})$ is defined as $\operatorname{rk}(\mathcal{A}) \triangleq \max \{\operatorname{deg}(x): x \in \mathcal{J}\}$. Let $\operatorname{dim}(\mathcal{J})$ denote the dimension of $\mathcal{J}$. Obviously, $\operatorname{rk}(\mathcal{A}) \leq \operatorname{dim}(\mathcal{J})$.

- An element $c \in \mathcal{J}$ is an idempotent if $c^{2}=c \neq 0$. An idempotent element is primitive if it cannot be written as a sum of two idempotents.

- A complete system of orthogonal idempotents in $\mathcal{A}$ is a finite $\operatorname{set}\left\{c_{1}, c_{2}, \ldots, c_{k}\right\}$ of idempotents where $c_{i} \circ c_{j}=0$ for all $i \neq j$, and $c_{1}+c_{2}+\cdots+c_{k}=e$.

- A Jordan frame in $\mathcal{A}$ is a complete system of orthogonal primitive idempotents. The number of elements of any Jordan frame equals the positive integer $\operatorname{rk}(\mathcal{A})$.

Example 2.1. Let $\mathbb{R}^{n}$ denote the space of $n$-dimensional real column vectors, and $\mathbb{R}_{+}^{n}$ be the nonnegative orthant. Consider $\mathbb{R}^{n}$ with the (usual) inner product and Jordan product defined, respectively, by $\langle x, y\rangle:=\sum_{i=1}^{n} x_{i} y_{i}$ and $x \circ y:=x * y$, where $x_{i}$ denotes the $i$ th component of $x$, etc., and $x * y$ denotes the componentwise product of vectors $x$ and $y$. Then $\left(\mathbb{R}^{n},\langle\cdot, \cdot\rangle, *\right)$ forms a Euclidean Jordan algebra with $\operatorname{rk}\left(\left(\mathbb{R}^{n},\langle\cdot, \cdot\rangle, *\right)\right)=\operatorname{dim}\left(\mathbb{R}^{n}\right)=n$ and $\mathbb{R}_{+}^{n}$ as its cone of squares. The identity element is the $n$-vector of ones, and the set $\left\{e_{1}, e_{2}, \ldots, e_{n}\right\}$ is the unique Jordan frame where $e_{i}$ is the $i$ th coordinate vector for $i \in\{1,2, \ldots, n\}$.

Example 2.2. Consider $\mathbb{R}^{n}(n \geq 2)$ where any $x$ is written as $x=\left(x_{1}, x_{2}^{T}\right)^{T}$ with $x_{1} \in \mathbb{R}$ and $x_{2} \in \mathbb{R}^{n-1}$. The inner product is the same as usual, and the Jordan product is defined by

$$
x \circ y=\left(\begin{array}{l}
x_{1} \\
x_{2}
\end{array}\right) \circ\left(\begin{array}{l}
y_{1} \\
y_{2}
\end{array}\right):=\left(\begin{array}{c}
\langle x, y\rangle \\
x_{1} y_{2}+y_{1} x_{2}
\end{array}\right) .
$$

Then $\Lambda^{n}:=\left(\mathbb{R}^{n},\langle\cdot, \cdot\rangle, \circ\right)$ forms a Euclidean Jordan algebra, and its cone of squares (Lorentz cone or second-order cone) is specified by $\Lambda_{+}^{n}:=\left\{\left(x_{1}, x_{2}^{T}\right)^{T}: x_{1} \geq\left\|x_{2}\right\|\right\}$, where $\|\cdot\|$ denotes the 2-norm. The identity element in $\Lambda^{n}$ is $e=\left(\begin{array}{l}1 \\ 0\end{array}\right)$. The set $\left\{c_{1}, c_{2}\right\}$ is a Jordan frame given by $c_{i}=\frac{1}{2}\left(\begin{array}{c}1 \\ (-1)^{i} \omega\end{array}\right)$ for $i=1,2$ with any $\omega \in \mathbb{R}^{n-1}$ satisfying $\|\omega\|=1$.

Example 2.3. Let $\mathbb{S}^{n}$ denote the set of all $n \times n$ real symmetric matrices with the inner product and Jordan product defined, respectively, by $\langle X, Y\rangle:=\operatorname{Trace}(X Y)$ and $X \circ Y:=(X Y+Y X) / 2$. Thus $\left(\mathbb{S}^{n},\langle\cdot, \cdot\rangle, \circ\right)$ forms a Euclidean Jordan algebra, and its cone of squares $\mathbb{S}_{+}^{n}$ is the set of all positive semidefinite symmetric matrices. The identity element in this setting is the identity matrix $E$. The set $\left\{E_{1}, E_{2}, \ldots, E_{n}\right\}$ is a Jordan frame where $E_{i}$ is the diagonal matrix with one in the $i i$-entry and zeros elsewhere for $i \in\{1,2, \ldots, n\}$.

We now review the following spectral decomposition theorem of an element in a Euclidean Jordan algebra.

Theorem 2.4. (Spectral Decomposition Type II (Theorem III.1.2, [10])) Let $\mathcal{A}$ be a Euclidean Jordan algebra with rank $r$. Then for $x \in \mathcal{J}$ there exist a Jordan frame $\left\{c_{1}, c_{2}, \ldots, c_{r}\right\}$ and real numbers $\lambda_{1}(x), \lambda_{2}(x), \ldots, \lambda_{r}(x)$ such that

$$
x=\lambda_{1}(x) c_{1}+\lambda_{2}(x) c_{2}+\cdots+\lambda_{r}(x) c_{r} .
$$

The numbers $\lambda_{i}(x)(i=1,2, \ldots, r)$ are the eigenvalues of $x$. We call (2.1) the spectral decomposition (or the spectral expansion) of $x$.

Note that the Jordan frame $\left\{c_{1}, c_{2}, \ldots, c_{r}\right\}$ in (2.1) depends on $x$. We do not write this dependence explicitly for simplicity of notation. (The same for $\left\{b_{1}, b_{2}, \ldots, b_{\bar{r}}\right\}$ 
below.) Let $\sigma(x)$ be the set of all distinct eigenvalues of $x$. Then $\sigma(x)$ contains at least one element and at most $r$. For each $\mu_{i}(x) \in \sigma(x)$, denote $N_{i}(x):=\left\{j: \lambda_{j}(x)=\right.$ $\left.\mu_{i}(x)\right\}$ and $b_{i} \triangleq \sum_{j \in N_{i}(x)} c_{j}$. Then the set $\left\{b_{i}: \mu_{i}(x) \in \sigma(x)\right\}$ is a complete system of orthogonal idempotents, and its uniqueness is guaranteed by Theorem III.1.1 in [10]. Let $\bar{r}$ be the number of elements in this set. We then have the spectral decomposition of type I stated in [10]; i.e.,

$$
x=\mu_{1}(x) b_{1}+\mu_{2}(x) b_{2}+\cdots+\mu_{\bar{r}}(x) b_{\bar{r}} .
$$

Next, we recall the Peirce decomposition theorem on the space $\mathcal{J}$, where a Jordan frame $\left\{c_{1}, c_{2}, \ldots, c_{r}\right\}$ is fixed beforehand. In this case, define the following subspaces

$$
J_{i i} \triangleq\left\{x \in \mathcal{J}: x \circ c_{i}=x\right\} \text { and } J_{i j} \triangleq\left\{x \in \mathcal{J}: x \circ c_{i}=\frac{1}{2} x=x \circ c_{j}\right\}, i \neq j,
$$

for $i, j \in\{1,2, \ldots, r\}$. In the second-order cone (SOC) case, we have $J_{12} \triangleq\left\{x \in \mathbb{R}^{n}\right.$ : $\left.x_{1}=0,\left\langle x_{2}, w\right\rangle=0\right\}$, where $w$ is characterized by the Jordan frame as in Example 2.2.

Theorem 2.5 (Theorem IV.2.1, [10]). Let $\left\{c_{1}, c_{2}, \ldots, c_{r}\right\}$ be a given Jordan frame in a Euclidean Jordan algebra $\mathcal{A}$ of rank $r$. Then $\mathcal{J}$ is the orthogonal direct sum of spaces $J_{i j}(i \leq j)$. Furthermore,

(i) $J_{i j} \circ J_{i j} \subseteq J_{i i}+J_{j j}$;

(ii) $J_{i j} \circ J_{j k} \subseteq J_{i k}$, if $i \neq k$;

(iii) $J_{i j} \circ J_{k l}=\{0\}$, if $\{i, j\} \cap\{k, l\}=\varnothing$.

For each $x \in \mathcal{J}$, we define the Lyapunov transformation $L(x): \mathcal{J} \rightarrow \mathcal{J}$ by $L(x) y=$ $x \circ y$ for all $y \in \mathcal{J}$, which is a symmetric operator in the sense that $\langle L(x) y, z\rangle=$ $\langle y, L(x) z\rangle$ for all $y, z \in \mathcal{J}$. Meanwhile, the operator $Q(x) \triangleq 2 L^{2}(x)-L\left(x^{2}\right)$ is called the quadratic representation of $x$. We say two elements $x, y \in \mathcal{J}$ operator commute if $L(x) L(y)=L(y) L(x)$. Lemma X.2.2 in [10] gives the following characterization of operator commutativity.

TheOREM 2.6. Two elements $x$ and $y$ of a Euclidean Jordan algebra of rank $r$ are operator commute if and only if they share a common Jordan frame.

Thus, for a given Jordan frame $\left\{c_{1}, c_{2}, \ldots, c_{r}\right\}$, it is easy to see that $c_{i}, c_{j}$ operator commute and $L\left(c_{i}\right) L\left(c_{j}\right)=L\left(c_{j}\right) L\left(c_{i}\right)$ for any $i, j \in\{1,2, \ldots, r\}$. So do $b_{i}$ and $b_{j}$ for any $i, j \in\{1,2, \ldots, \bar{r}\}$.

2.2. The Jacobian of Löwner operators. We review differentiability and semismoothness of a vector-valued function, which was called the Löwner operator by Sun and Sun [30] in recognition of Löwner's contribution [21]. We also present some new results on the Jacobian and the Clarke generalized Jacobian of the Löwner operator, which are basic and useful in the subsequent analysis.

Definition 2.7. Let $x=\sum_{j=1}^{r} \lambda_{j}(x) c_{j}$ and $g: \mathbb{R} \rightarrow \mathbb{R}$ be a real-valued function. We define the Löwner operator (function) $G: \mathcal{J} \rightarrow \mathcal{J}$ as

$$
G(x) \triangleq \sum_{j=1}^{r} g\left(\lambda_{j}(x)\right) c_{j}=g\left(\lambda_{1}(x)\right) c_{1}+g\left(\lambda_{2}(x)\right) c_{2}+\cdots+g\left(\lambda_{r}(x)\right) c_{r} .
$$

When $g(t)=t_{+}=\max \{0, t\}(t \in \mathbb{R})$, this becomes the metric projection operator

$$
P_{K}(x) \triangleq\left(\lambda_{1}(x)\right)_{+} c_{1}+\left(\lambda_{2}(x)\right)_{+} c_{2}+\cdots+\left(\lambda_{r}(x)\right)_{+} c_{r}
$$

onto the symmetric cone $K$. Note that $x \in K$ (respectively, $x \in \operatorname{int} K$ ) if and only if $\lambda_{i}(x) \geq 0$ (respectively, $\left.\lambda_{i}(x)>0\right)(i=1,2, \ldots, r)$. For any $x \in K$, we define $\sqrt{x} \triangleq \sum_{j=1}^{r} \sqrt{\lambda_{j}(x)} c_{j}$ for $x \in K$. 
We consider the differentiability of the Löwner operator $G(\cdot)$. Suppose that $g$ is differentiable at $\tau_{i}, i=1,2, \ldots, r$. Define the first divided difference $g^{[1]}(\tau)$ of $g$ at $\tau \triangleq\left(\tau_{1}, \tau_{2}, \ldots, \tau_{r}\right)^{T} \in \mathbb{R}^{r}$ as the $r \times r$ symmetric matrix with the $i j$ th entry given by

$$
\left[g^{[1]}(\tau)\right]_{i j}=\left[\tau_{i}, \tau_{j}\right]_{g} \triangleq\left\{\begin{array}{cl}
\frac{g\left(\tau_{i}\right)-g\left(\tau_{j}\right)}{\tau_{i}-\tau_{j}} & \text { if } \tau_{i} \neq \tau_{j}, \quad i, j=1,2, \ldots, r . \\
g^{\prime}\left(\tau_{i}\right) & \text { if } \tau_{i}=\tau_{j},
\end{array}\right.
$$

A direct implication of Theorem 3.2 in [30] is the following property of the Jacobian of the Löwner operator $G(\cdot)$.

THEOREM 2.8. Let $x=\sum_{j=1}^{r} \lambda_{j}(x) c_{j}=\sum_{i=1}^{\bar{r}} \mu_{i}(x) b_{i}$. Then, $G(\cdot)$ is (continuously) differentiable at $x$ if and only if for each $j \in\{1,2, \ldots, r\}, g$ is (continuously) differentiable at $\lambda_{j}(x)$. In this case, the Jacobian $\nabla G(x)$ is given by

$$
\nabla G(x)=2 \sum_{i \neq j, i, j=1}^{r}\left[\lambda_{i}(x), \lambda_{j}(x)\right]_{g} L\left(c_{i}\right) L\left(c_{j}\right)+\sum_{i=1}^{r} g^{\prime}\left(\lambda_{i}(x)\right) Q\left(c_{i}\right)
$$

or equivalently

$$
\nabla G(x)=2 \sum_{i \neq j, i, j=1}^{\bar{r}}\left[\mu_{i}(x), \mu_{j}(x)\right]_{g} L\left(b_{i}\right) L\left(b_{j}\right)+\sum_{i=1}^{\bar{r}} g^{\prime}\left(\mu_{i}(x)\right) Q\left(b_{i}\right) .
$$

Furthermore, $\nabla G(x)$ is a linear and symmetric operator from $\mathcal{J}$ into itself.

As a consequence of Theorem 2.8, we obtain the following result in the case of $\operatorname{rk}(\mathcal{A})=\operatorname{dim}(\mathcal{J})$.

Corollary 2.9. Suppose that $\operatorname{rk}(\mathcal{A})=\operatorname{dim}(\mathcal{J})=n$ and $x=\sum_{j=1}^{n} \lambda_{j}(x) c_{j}=$ $\sum_{i=1}^{\bar{n}} \mu_{i}(x) b_{i}$. If $G(\cdot)$ is (continuously) differentiable at $x$, then it holds that

$$
\nabla G(x)=\sum_{i=1}^{n} g^{\prime}\left(\lambda_{i}(x)\right) L\left(c_{i}\right)=\sum_{i=1}^{\bar{n}} g^{\prime}\left(\mu_{i}(x)\right) L\left(b_{i}\right) .
$$

Proof. Since $\operatorname{rk}(\mathcal{A})=\operatorname{dim}(\mathcal{J})=n$, it follows from Theorem 3.5 in [20] that there is a unique Jordan frame $\left\{c_{1}, c_{2}, \ldots, c_{n}\right\}$ in $\mathcal{A}$. Thus, through Theorem 2.5, any element $h \in \mathcal{J}$ can be expressed by $h=\sum_{i=1}^{n} h_{i} c_{i}$ with $h_{i} \in \mathbb{R}(i=1,2, \ldots, n)$. Therefore,

$$
L\left(c_{i}\right) L\left(c_{j}\right) h=L\left(c_{j}\right) L\left(c_{i}\right) h=c_{i} \circ\left(c_{j} \circ h\right)= \begin{cases}c_{i} \circ\left(h_{j} c_{j}\right)=0 & \text { if } i \neq j, \\ c_{i} \circ\left(h_{i} c_{i}\right)=c_{i} \circ h & \text { if } i=j .\end{cases}
$$

This implies that $L\left(c_{i}\right) L\left(c_{j}\right)=0(i \neq j)$ and $L\left(c_{i}\right) L\left(c_{i}\right)=L\left(c_{i}\right)$ for any $i, j \in$ $\{1,2, \ldots, n\}$. Hence $Q\left(c_{i}\right)=L\left(c_{i}\right)$. Formula $(2.8)$ is then an implication of Theorem 2.8 .

As an application of Corollary 2.9, we consider the Jacobian of the Löwner operator on $\mathbb{R}^{n}$.

Example 2.10. Suppose that $\mathcal{A}=\left(\mathbb{R}^{n},\langle\cdot, \cdot\rangle, *\right)$ as in Example 2.1. Let $x=$ $\sum_{i=1}^{n} x_{i} e_{i}$. One can easily verify that $L\left(e_{i}\right)=e_{i} e_{i}^{T}=E_{i}(i=1,2, \ldots, n)$. Note that $\operatorname{rk}\left(\left(\mathbb{R}^{n},\langle\cdot, \cdot\rangle, *\right)\right)=\operatorname{dim}\left(\mathbb{R}^{n}\right)=n$. It is obvious via Corollary 2.9 that

$$
\nabla G(x)=\sum_{i=1}^{n} g^{\prime}\left(x_{i}\right) L\left(e_{i}\right)=\operatorname{Diag}\left\{g^{\prime}\left(x_{1}\right), g^{\prime}\left(x_{2}\right), \ldots, g^{\prime}\left(x_{n}\right)\right\} .
$$

Copyright $@$ by SIAM. Unauthorized reproduction of this article is prohibited. 
The next theorem presents a sufficient condition which guarantees that the Jacobian $\nabla G(x)$ is positive semidefinite (respectively, positive definite). Here $\nabla G(x)$ is called positive semidefinite (respectively, positive definite) if $\langle h, \nabla G(x) h\rangle \geq 0$ for all $h \in \mathcal{J}$ (respectively, $\langle h, \nabla G(x) h\rangle>0$ for all $0 \neq h \in \mathcal{J}$ ).

THEOREM 2.11. Let $x=\sum_{j=1}^{r} \lambda_{j}(x) c_{j}$. If $g$ is (continuously) differentiable at $\lambda_{j}(x)$ for each $j \in\{1,2, \ldots, r\}$ and $g^{\prime}(t) \geq 0$ for all $t \in \mathbb{R}$, then $G(\cdot)$ is (continuously) differentiable at $x$ and the Jacobian $\nabla G(x)$ is positive semidefinite. Moreover, the Jacobian is positive definite if the condition $g^{\prime}(t) \geq 0$ is replaced by $g^{\prime}(t)>0$.

Proof. Let $x=\sum_{j=1}^{r} \lambda_{j}(x) c_{j}$. By Theorem 2.5, any element $h \in \mathcal{J}$ can be expressed by $h=\sum_{i=1}^{r} h_{i} c_{i}+\sum_{1 \leq k<l \leq r} h_{k l}$ where $h_{i} \in \mathbb{R}(i=1,2, \ldots, r)$ and $h_{k l} \in$ $J_{k l}(1 \leq k<l \leq r)$. Theorem 2.5 also implies that $c_{j} \circ \sum_{i=1}^{r} h_{i} c_{i}=h_{j} c_{j}$ and $c_{j} \circ \sum_{1 \leq k<l \leq r} h_{k l}=\frac{1}{2}\left(\sum_{k<j} h_{k j}+\sum_{l>j} h_{j l}\right)$. It therefore holds that

$$
c_{j} \circ h=h_{j} c_{j}+\frac{1}{2}\left(\sum_{k=1}^{j-1} h_{k j}+\sum_{l=j+1}^{r} h_{j l}\right),
$$

where $\sum_{k=1}^{j-1} h_{k j} \triangleq 0$ if $j=1$ and $\sum_{l=j+1}^{r} h_{j l} \triangleq 0$ if $j=r$. Furthermore, Theorem 2.5 implies that

$$
\left\langle h, c_{j} \circ\left(c_{i} \circ h\right)\right\rangle=\left\langle c_{j} \circ h, c_{i} \circ h\right\rangle=\frac{1}{4}\left\langle h_{j i}, h_{j i}\right\rangle=\frac{1}{4}\left\|h_{j i}\right\|^{2}, \forall j \neq i,
$$

and

$$
Q\left(c_{j}\right) h=2 c_{j} \circ\left(c_{j} \circ h\right)-c_{j} \circ h=h_{j} c_{j}, \quad j=1,2, \ldots, r .
$$

Meanwhile, noting that $c_{j}^{2}=c_{j}$, one has $\left\langle h, c_{j}\right\rangle=\left\langle c_{j} \circ h, c_{j}\right\rangle=h_{j}\left\langle c_{j}, c_{j}\right\rangle=h_{j}\left\|c_{j}\right\|^{2}$. Combining this with (2.6), (2.10), and (2.11) and noting that $L\left(c_{j}\right) L\left(c_{i}\right) h=c_{j} \circ\left(c_{i} \circ h\right)$, one has

$$
\begin{aligned}
\langle h, \nabla G(x) h\rangle & =\left\langle h, \sum_{j \neq i, j, i=1}^{r} 2\left(g^{[1]}(\lambda(x))\right)_{j i} c_{j} \circ\left(c_{i} \circ h\right)+\sum_{j=1}^{r}\left(g^{[1]}(\lambda(x))\right)_{j j} h_{j} c_{j}\right\rangle \\
& =\sum_{j \neq i, j, i=1}^{r} 2\left(g^{[1]}(\lambda(x))\right)_{j i}\left\langle h, c_{j} \circ\left(c_{i} \circ h\right)\right\rangle+\sum_{j=1}^{r}\left(g^{[1]}(\lambda(x))\right)_{j j} h_{j}\left\langle h, c_{j}\right\rangle \\
& =\frac{1}{2} \sum_{j \neq i, j, i=1}^{r}\left(g^{[1]}(\lambda(x))\right)_{j i}\left\|h_{j i}\right\|^{2}+\sum_{j=1}^{r}\left(g^{[1]}(\lambda(x))\right)_{j j} h_{j}^{2}\left\|c_{j}\right\|^{2} .
\end{aligned}
$$

If $g^{\prime}(t) \geq 0\left(g^{\prime}(t)>0\right)$ for all $t \in \mathbb{R}$, then by (2.5) we can easily get $\left(g^{[1]}(\lambda(x))\right)_{j i} \geq 0$ $\left(\left(g^{[1]}(\lambda(x))\right)_{j i}>0\right)$ for all $j \neq i, j, i=1,2, \ldots, r$. Hence, $\langle h, \nabla G(x) h\rangle \geq 0$ for all $h \in \mathcal{J}(\langle h, \nabla G(x) h\rangle>0$ for all $0 \neq h \in \mathcal{J})$ through the above equation.

We proceed to study the semismoothness of the Löwner operator $G(\cdot)$. Semismoothness was originally introduced by Mifflin [24] for functionals. Qi and Sun [26] extended the concept of semismoothness to vector-valued functions and developed a systematic theory that employs semismoothness in the analysis of the superlinear convergence of Newton methods for solving systems of nondifferentiable equations.

We briefly review some concepts and results of the semismoothness from [26]. Let $\digamma: C \subseteq \mathcal{X} \rightarrow \mathcal{Y}$ be a locally Lipschitz function on an open set $C$. By Rademacher's theorem, $\digamma$ is almost everywhere differentiable (in the sense of Fréchet) in $C$. Let $D_{\digamma}$

Copyright $@$ by SIAM. Unauthorized reproduction of this article is prohibited. 
be the set of points where $\digamma$ is differentiable. Let $\digamma^{\prime}(x)$ denote the derivative of $\digamma$ at $x \in D_{\digamma}$ and let $\nabla \digamma(x)$ denote the Jacobian of $\digamma$ at $x$, which is the adjoint operator of $\digamma^{\prime}(x)$, in the sense of $\langle y, \nabla \digamma(x) z\rangle=\left\langle\digamma^{\prime}(x) y, z\right\rangle$ for all $y \in \mathcal{X}$ and $z \in \mathcal{Y}$. Then, the Clarke generalized Jacobian of $\digamma$ at $x$ is defined by $\partial \digamma(x) \triangleq \operatorname{conv}\left\{\partial_{B} \digamma(x)\right\}$, where $\partial_{B} \digamma(x) \triangleq\left\{\lim _{\bar{x} \rightarrow x, \bar{x} \in D_{\digamma}} \nabla \digamma(\bar{x})\right\}$. Observe that $\partial \digamma(x)=\{\nabla \digamma(x)\}$ if $\digamma$ is smooth (continuously differentiable) at $x$. We say $\digamma$ is directionally differentiable at $x$ along the direction $d$ if

$$
\digamma^{\prime}(x, d) \triangleq \lim _{t \downarrow 0} \frac{\digamma(x+t d)-\digamma(x)}{t} \quad \text { exists, }
$$

where $\digamma^{\prime}(x, d)$ is called the directional derivative of $\digamma$ at $x$ along the direction $d$; and $\digamma$ is directionally differentiable at $x$ if $\digamma$ is directionally differentiable at $x$ along any direction $d \neq 0$.

Employing the above concepts, we can define (strong) semismoothness of a function $\digamma$.

Definition 2.12. A directionally differentiable and locally Lipschitz function $\digamma: C \subseteq \mathcal{X} \rightarrow \mathcal{Y}$ is semismooth at $x \in C$ if $V^{T} d-\digamma^{\prime}(x ; d)=o(\|d\|)$ for any $d \neq 0, d \in \mathcal{X}$ sufficiently small and $V \in \partial \digamma(x+d)$. In particular, if $o(\|d\|)$ can be replaced by $O\left(\|d\|^{2}\right), \digamma$ is called strongly semismooth.

By combining Theorem 3.3 with Proposition 3.3 in [30], we have the following result on (strong) semismoothness of the Löwner operator $G(\cdot)$.

Lemma 2.13. Let $x=\sum_{j=1}^{r} \lambda_{j}(x) c_{j}$. Then $G(\cdot)$ is (strongly) semismooth at $x$ if and only if for each $j \in\{1,2, \ldots, r\}, g$ is (strongly) semismooth at $\lambda_{j}(x)$. In particular, the metric projection operator $P_{K}$ is strongly semismooth on $\mathcal{J}$.

We are ready to extend Theorems 2.8 and 2.11 to the case of a semismooth Löwner operator $G(\cdot)$. Let $g$ be semismooth at $\tau_{i}(i=1,2, \ldots, r)$ and $\partial g$ denote the generalized Jacobian of $g$ in the sense of Clarke. Define the first generalized divided difference $g^{[1, \partial]}$ of $g$ at $\tau$ as the set of all $r \times r$ symmetric matrices, where the $i j$ th entry $\left(g^{[1, \partial]}(\tau)\right)_{i j}$ of the element $g^{[1, \partial]}(\tau) \in g^{[1, \partial]}$ is given by a set $\left\{\left[\tau_{i}, \tau_{j}\right]_{g}\right\}$ for $i, j=1,2, \ldots, r$, where

$$
\left\{\left[\tau_{i}, \tau_{j}\right]_{g}\right\}=\left\{\begin{array}{cc}
\left\{\frac{g\left(\tau_{i}\right)-g\left(\tau_{j}\right)}{\tau_{i}-\tau_{j}}\right\} & \text { if } \tau_{i} \neq \tau_{j}, \\
\partial g\left(\tau_{i}\right) & \text { if } \tau_{i}=\tau_{j} .
\end{array}\right.
$$

Theorem 2.14. Let $x \in \mathcal{J}$. Then $G(\cdot)$ is (strongly) semismooth at $x$ if and only if $g$ is (strongly) semismooth at every eigenvalue of $x$. In this case, the Clarke generalized Jacobian $\partial G(x)$ satisfies

$$
\bar{\partial} G(x) \supseteq \partial G(x) \supseteq \underline{\partial} G(x)
$$

with the sets $\bar{\partial} G(x)$ and $\underline{\partial} G(x)$ being given, respectively, by

$$
\begin{aligned}
& \bar{\partial} G(x) \triangleq \operatorname{conv}\left[\bigcup_{\left\{c_{1}, \ldots, c_{r}\right\} \in \mathcal{C}(x)} \partial_{c_{1}, \ldots, c_{r}} G(x)\right], \\
& \underline{\partial} G(x) \triangleq\left\{2 \sum_{i \neq j, i, j=1}^{\bar{r}}\left[\mu_{i}(x), \mu_{j}(x)\right]_{g} L\left(b_{i}(x)\right) L\left(b_{j}\right)+\sum_{i=1}^{\bar{r}} \partial g\left(\mu_{i}(x)\right) Q\left(b_{i}\right)\right\},
\end{aligned}
$$

where $\mathcal{C}(x)$ is the set consisting of all Jordan frames in the spectral decomposition type 
II of $x$, and $\partial_{c_{1}, \ldots, c_{r}} G(x) \triangleq\left\{2 \sum_{i \neq j, i, j=1}^{r}\left\{\left[\lambda_{i}(x), \lambda_{j}(x)\right]_{g}\right\} L\left(c_{i}\right) L\left(c_{j}\right)+\sum_{i=1}^{r} \partial g\left(\lambda_{i}(x)\right)\right.$ $\left.Q\left(c_{i}\right)\right\}$.

Proof. The first part of the theorem follows from Lemma 2.13. For the second part, we first show $\bar{\partial} G(x) \supseteq \partial G(x)$. By the definitions of $\bar{\partial} G$ and $\partial G$ we need to prove only that $\bigcup_{\left\{c_{1}, \ldots, c_{r}\right\} \in \mathcal{C}(x)} \partial_{c_{1}, \ldots, c_{r}} G(x) \supseteq \partial_{B} G(x)$. Taking any $V \in \partial_{B} G(x)$, by the definition of $\partial_{B} G(x)$ there exists a vector $h \triangleq h(V) \in \mathcal{J}$ such that $V=$ $\lim _{h \rightarrow 0, x+h \in D_{G}} \nabla G(x+h)$. In order to show $V \in \bigcup_{\left\{c_{1}, \ldots, c_{r}\right\} \in \mathcal{C}(x)} \partial_{c_{1}, \ldots, c_{r}} G(x)$, we proceed as follows.

Take any $\left\{c_{1}, \ldots, c_{r}\right\} \in \mathcal{C}(x)$ and let $x=\sum_{j=1}^{r} \lambda_{j}(x) c_{j}=\sum_{i=1}^{\bar{r}} \mu_{i}(x) b_{i}(x)$ with $\lambda_{1}(x) \geq \lambda_{2}(x) \geq \cdots \geq \lambda_{r}(x)$ and $\mu_{1}(x)>\mu_{2}(x)>\cdots>\mu_{\bar{r}}(x)$. For the above $h \in \mathcal{J}$, let $x+h \triangleq \sum_{j=1}^{r} \lambda_{j}(x+h) c_{j}(x+h)$ with $\lambda_{1}(x+h) \geq \lambda_{2}(x+h) \geq \cdots \geq \lambda_{r}(x+h)$. By Theorem 2.4 and the argument after it, in the sense of set convergence (see, e.g., [27]), one has

$$
\lim _{h \rightarrow 0, x+h \in D_{G}}\{\lambda(x+h)\}=\{\lambda(x)\}
$$

where $\lambda(x+h) \triangleq\left(\lambda_{1}(x+h), \lambda_{2}(x+h), \ldots, \lambda_{r}(x+h)\right)^{T}$ and $\lambda(x) \triangleq\left(\lambda_{1}(x), \lambda_{2}(x), \ldots\right.$, $\left.\lambda_{r}(x)\right)^{T}$. Similarly, using "lim sup" in the sense of set convergence, we have

$$
\limsup _{h \rightarrow 0, x+h \in D_{G}}\left\{\left(c_{1}(x+h), c_{2}(x+h), \ldots, c_{r}(x+h)\right)\right\} \subseteq \mathcal{C}(x) .
$$

Notice that for any $i, j=1,2, \ldots, r$,

$$
\limsup _{h \rightarrow 0, x+h \in D_{G}}\left\{\left[\lambda_{i}(x+h), \lambda_{j}(x+h)\right]_{g}\right\}\left\{\begin{array}{cc}
=\left\{\frac{g\left(\lambda_{i}(x)\right)-g\left(\lambda_{j}(x)\right)}{\lambda_{i}(x)-\lambda_{j}(x)}\right\} & \text { if } \lambda_{i}(x) \neq \lambda_{j}(x), \\
\subseteq \partial g\left(\lambda_{i}(x)\right) & \text { if } \lambda_{i}(x)=\lambda_{j}(x) .
\end{array}\right.
$$

Thus,

$$
\limsup _{h \rightarrow 0, x+h \in D_{G}}\left\{\left[\lambda_{i}(x+h), \lambda_{j}(x+h)\right]_{g}\right\} \subseteq\left\{\left[\lambda_{i}(x), \lambda_{j}(x)\right]_{g}\right\} .
$$

Also, it holds by (2.6) that for $x+h \in D_{G}$,

$$
\begin{aligned}
\nabla G(x+h)= & 2 \sum_{i \neq j, i, j=1}^{r}\left[\lambda_{i}(x+h), \lambda_{j}(x+h)\right]_{g} L\left(c_{i}(x+h)\right) L\left(c_{j}(x+h)\right) \\
& +\sum_{i=1}^{r} g^{\prime}\left(\lambda_{i}(x+h)\right) Q\left(c_{i}(x+h)\right) .
\end{aligned}
$$

This, together with (2.12), (2.13), and the continuity property of $L(x)$ and $Q(x)$, leads to

$$
\begin{aligned}
& \limsup _{h \rightarrow 0, x+h \in D_{G}}\{\nabla G(x+h)\} \\
\subseteq & \bigcup_{\left\{c_{1}, \ldots, c_{r}\right\} \in \mathcal{C}(x)}\left\{2 \sum_{i \neq j, i, j=1}^{r}\left\{\left[\lambda_{i}(x), \lambda_{j}(x)\right]_{g}\right\} L\left(c_{i}\right) L\left(c_{j}\right)+\sum_{i=1}^{r} \partial g\left(\lambda_{i}(x)\right) Q\left(c_{i}\right)\right\} .
\end{aligned}
$$

Clearly, $V=\lim _{h \rightarrow 0, x+h \in D_{G}} \nabla G(x+h) \in \limsup _{h \rightarrow 0, x+h \in D_{G}}\{\nabla G(x+h)\}$. This implies that $V \in \bigcup_{\left\{c_{1}, \ldots, c_{r}\right\} \in \mathcal{C}(x)} \partial_{c_{1}, \ldots, c_{r}} G(x)$ by the definition of $\partial_{c_{1}, \ldots, c_{r}} G(x)$. 
We next prove $\partial G(x) \supseteq \underline{\partial} G(x)$. For any $W(x) \in \underline{\partial} G(x)$ with $x=\sum_{i=1}^{\bar{r}} \mu_{i}(x) b_{i}(x)$ and $\mu_{1}(x)>\mu_{2}(x)>\cdots>\mu_{\bar{r}}(x)$, by the definition of $\underline{\partial} G(x)$ we have

$$
W(x)=2 \sum_{i \neq j, i, j=1}^{\bar{r}}\left[\mu_{i}(x), \mu_{j}(x)\right]_{g} L\left(b_{i}(x)\right) L\left(b_{j}(x)\right)+\sum_{i=1}^{\bar{r}} w_{i} Q\left(b_{i}(x)\right)
$$

with $w_{i} \in \partial g\left(\mu_{i}(x)\right)(i=1,2, \ldots, \bar{r})$. Since $g$ is semismooth at $\mu_{i}(x), \partial g\left(\mu_{i}(x)\right)$ and $\partial_{B} g\left(\mu_{i}(x)\right)$ are well-defined and $\operatorname{dim}\left(\partial g\left(\mu_{i}(x)\right)\right)=1$. Let $D_{g}$ be the set consisting of all the differentiable points of $g$. By Carathéodory theorem (see [27]), for any given $w_{i} \in \partial g\left(\mu_{i}(x)\right)$ there exist $t_{i} \in[0,1]$ and two subsequences $\left\{h_{i, 0}\right\}$ and $\left\{h_{i, 1}\right\}$ converging to 0 with $\mu_{i}(x)+h_{i, 0}, \mu_{i}(x)+h_{i, 1} \in D_{g}$ such that

$$
w_{i, l_{i}} \triangleq \lim _{h_{i, l_{i} \rightarrow 0, \mu_{i}(x)+h_{i, l_{i}} \in D_{g}}} g^{\prime}\left(\mu_{i}(x)+h_{i, l_{i}}\right) \in \partial_{B} g\left(\mu_{i}(x)\right), \quad l_{i} \in\{0,1\}
$$

and

$$
w_{i}=t_{i} w_{i, 0}+\left(1-t_{i}\right) w_{i, 1} .
$$

Based on the set $\left\{h_{i, l_{i}}: l_{i} \in\{0,1\}, i=1,2, \ldots, \bar{r}\right\}$, we construct a set $\mathcal{H}$ by

$$
\mathcal{H} \triangleq\left\{\sum_{i=1}^{\bar{r}} h_{i, l_{i}} b_{i}(x): l_{i} \in\{0,1\}\right\} .
$$

Let $l \triangleq\left(l_{1}, l_{2}, \ldots, l_{\bar{r}}\right)$ and $h_{l} \triangleq \sum_{i=1}^{\bar{r}} h_{i, l_{i}} b_{i}(x)$ with $l_{i} \in\{0,1\}$. Then the set $\mathcal{H}$ can be rewritten as $\mathcal{H} \triangleq\left\{h_{l}: l \in\{0,1\}^{\bar{r}}\right\}$, which includes $2^{\bar{r}}$ elements. Meanwhile, for each element $h_{l}$, we have

$$
x+h_{l}=\sum_{i=1}^{\bar{r}}\left(\mu_{i}(x)+h_{i, l_{i}}\right) b_{i}(x) .
$$

Moreover, taking sufficiently small $\left\|h_{l}\right\|$, we have $\mu_{1}(x)+h_{1, l_{1}}>\mu_{2}(x)+h_{2, l_{2}}>\cdots>$ $\mu_{\bar{r}}(x)+h_{\bar{r}, l_{\bar{r}}}$, and hence $\mu_{i}\left(x+h_{l}\right)=\mu_{i}(x)+h_{i, l_{i}}, b_{i}\left(x+h_{l}\right)=b_{i}(x)$ by the uniqueness of spectral decomposition type I. Thus, $x+h_{l} \in D_{G}$ by $\mu_{i}(x)+h_{i, l_{i}} \in D_{g}$, and from (2.7) and (2.14) we obtain

$$
\begin{gathered}
W_{l}(x) \triangleq \lim _{h_{l} \rightarrow 0, x+h_{l} \in D_{G}} \nabla G\left(x+h_{l}\right) \\
=2 \lim _{h_{l} \rightarrow 0, x+h_{l} \in D_{G}}\left[\sum_{i \neq j, i, j=1}^{\bar{r}}\left[\mu_{i}(x)+h_{i, l_{i}}, \mu_{j}(x)+h_{j, l_{j}}\right]_{g} L\left(b_{i}(x)\right) L\left(b_{j}(x)\right)\right. \\
\left.\quad+\sum_{i=1}^{\bar{r}} g^{\prime}\left(\mu_{i}(x)+h_{i, l_{i}}\right) Q\left(b_{i}(x)\right)\right] \\
=2 \sum_{i \neq j, i, j=1}^{\bar{r}}\left[\mu_{i}(x), \mu_{j}(x)\right]_{g} L\left(b_{i}(x)\right) L\left(b_{j}(x)\right)+\sum_{i=1}^{\bar{r}} w_{i, l_{i}} Q\left(b_{i}(x)\right) .
\end{gathered}
$$

Therefore, $W_{l}(x) \in \partial_{B} G(x)$ for every $l \in\{0,1\}^{\bar{r}}$. This implies that

$$
\mathcal{W}(x) \triangleq \operatorname{conv}\left\{W_{l}(x): l \in\{0,1\}^{\bar{r}}\right\} \subseteq \partial G(x) .
$$

Copyright (c) by SIAM. Unauthorized reproduction of this article is prohibited. 
To prove $W(x) \in \partial G(x)$, it suffices to claim that $W(x) \in \mathcal{W}(x)$. In fact, from expressions of $W(x)$ and $W_{l}(x)$, it is easy to see that $w \triangleq\left(w_{1}, w_{2}, \ldots, w_{\bar{r}}\right)$ given above lies in the hypercube whose extreme points are defined by $w_{i, l_{i}}$ with $l_{i} \in\{0,1\}, i=$ $1,2, \ldots, \bar{r}$. Hence, $W(x)$ must be a convex combination of points $\left\{W_{l}(x): l \in\right.$ $\left.\{0,1\}^{\bar{r}}\right\}$. The proof is completed.

Remark 2.15. From Theorem 2.14, we easily observe that if $x \in \mathcal{J}$ has distinct eigenvalues $\lambda_{1}(x), \ldots, \lambda_{r}(x)$ and $\mathcal{C}(x)$ has an element, then $\underline{\partial} G(x)=\partial G(x)=\bar{\partial} G(x)$. However, if $x$ has the multiple eigenvalues or $\mathcal{C}(x)$ contains many elements, the sets $\underline{\partial} G(x), \partial G(x)$, and $\bar{\partial} G(x)$ may be different as the following example shows.

Let $\mathcal{A}=\Lambda^{n}(n \geq 3)$ and $x=\sum_{i=1}^{2} \lambda_{i} c_{i}$ as in Example 2.2. Take $G(x)=$ $P_{K}(x)$ where $g(t)=t_{+}$, and let $x=0$. Then $\lambda_{1}=\lambda_{2}=0$, and there are infinitely many Jordan frames at $x=0$. The direct calculation yields $\bar{\partial} P_{\Lambda_{+}^{n}}(0)=$ $\operatorname{conv}\left\{4[0,1] L\left(c_{1}\right) L\left(c_{2}\right)+[0,1] Q\left(c_{1}\right)+[0,1] Q\left(c_{2}\right)\right\}$ and $\underline{\partial} P_{\Lambda_{+}^{n}}(0)=\operatorname{conv}\{0, E\}$ where $\operatorname{conv}\{0, E\}=\{\alpha E: 0 \leq \alpha \leq 1\}$. Note that $\partial P_{\Lambda_{+}^{n}}(0)=\operatorname{conv}\{0, E, S\}$ by Proposition 4.8 in [15] where $S$ satisfies

$$
S=4 \times \frac{1+\beta}{2} L\left(c_{1}\right) L\left(c_{2}\right)+0 \times Q\left(c_{1}\right)+Q\left(c_{2}\right),
$$

where $\frac{1+\beta}{2} \in[0,1]$. A simple calculation checks that $\underline{\partial} P_{\Lambda_{+}^{n}}(0) \subset \partial P_{\Lambda_{+}^{n}}(0) \subset$ $\bar{\partial} P_{\Lambda_{+}^{n}}(0)$.

Remark 2.16. Suppose that $\operatorname{rk}(\mathcal{A})=\operatorname{dim}(\mathcal{J})=n$ and $x=\sum_{j=1}^{n} \lambda_{j}(x) c_{j}=$ $\sum_{i=1}^{\bar{n}} \mu_{i}(x) b_{i}$ as in the case of Corollary 2.9. If $G(\cdot)$ is (strongly) semismooth at $x$, we derive by Theorem 2.14 that $\underline{\partial} G(x) \subseteq \partial G(x) \subseteq \bar{\partial} G(x)$, where

$$
\bar{\partial} G(x)=\sum_{i=1}^{n} \partial g\left(\lambda_{i}(x)\right) L\left(c_{i}\right), \quad \underline{\partial} G(x)=\sum_{i=1}^{\bar{n}} \partial g\left(\mu_{i}(x)\right) L\left(b_{i}\right) .
$$

Especially, when $\mathcal{A}=\left(\mathbb{R}^{n},\langle\cdot, \cdot\rangle, *\right)$ as in Example 2.10 and $x=\sum_{i=1}^{n} x_{i} e_{i}=\sum_{i=1}^{\bar{n}} y_{i}$ $\left(\sum_{j \in N(i)} e_{j}\right)$, in the similar way to the second part in the proceeding proof, one has $\bar{\partial} G(x) \subseteq \partial G(x)$. Hence,

$$
\begin{aligned}
& \bar{\partial} G(x)=\partial G(x)=\sum_{i=1}^{n} \partial g\left(x_{i}\right) E_{i}=\operatorname{Diag}\left\{\partial g\left(x_{1}\right), \ldots, \partial g\left(x_{n}\right)\right\}, \\
& \underline{\partial} G(x)=\sum_{i=1}^{\bar{n}} \partial g\left(y_{i}\right)\left(\sum_{j \in N(i)} E_{j}\right)=\operatorname{Diag}\left\{\partial g\left(y_{1}\right) I_{1}, \ldots, \partial g\left(y_{\bar{n}}\right) I_{\bar{n}}\right\},
\end{aligned}
$$

where $I_{i}$ is the $|N(i)| \times|N(i)|$ identity matrix for $i=1,2, \ldots, \bar{n}$. Moreover, letting $G(x)=P_{K}(x)$ and $x=0$, we derive

$$
\begin{aligned}
& \bar{\partial} P_{\mathbb{R}_{+}^{n}}(0)=\partial P_{\mathbb{R}_{+}^{n}}(0)=\left\{\left(\begin{array}{cccc}
{[0,1]} & 0 & \cdots & 0 \\
0 & {[0,1]} & \cdots & 0 \\
\vdots & \vdots & \ddots & \vdots \\
0 & 0 & \cdots & {[0,1]}
\end{array}\right)\right\} \\
& \supset\{\alpha E, 0 \leq \alpha \leq 1\}=\underline{\partial} P_{\mathbb{R}_{+}^{n}}(0) .
\end{aligned}
$$

Theorem 2.14 provides an approximation to the Clarke generalized Jacobian, which can be successfully employed to prove the positive semidefiniteness of $\partial G(\cdot)$. 
THEOREM 2.17. If $g$ is (strongly) semismooth at every eigenvalue of $x$ and $\partial g(t) \subseteq \mathbb{R}_{+}\left(\partial g(t) \subseteq \mathbb{R}_{++}\right)$for all $t \in \mathbb{R}$, then the function $G(\cdot)$ is (strongly) semismooth at $x$, and all the element $V \in \partial G(x)$ are positive semidefinite (positive definite). Moreover, when $\partial g(t) \subseteq \mathbb{R}_{++}$, there exists a scalar $\alpha(x)>0$ such that $V \succeq \alpha(x) I \succ 0$.

Proof. By Theorem 2.14 and the definition of $\bar{\partial} G$, it suffices to demonstrate that if $\partial g(t) \subseteq \mathbb{R}_{++}$for all $t \in \mathbb{R}$, then for any $\left\{c_{1}, \ldots, c_{r}\right\} \in \mathcal{C}(x)$ and $V \in \partial_{c_{1}, \ldots, c_{r}} G(x)$ there is a scalar $\alpha(x)$ such that $V \succeq \alpha(x) I \succ 0$. In this case, one has $x=\sum_{i=1}^{r} \lambda_{i}(x) c_{i}$ and

$$
V=2 \sum_{i \neq j, i, j=1}^{r} \nu_{i j}(x) L\left(c_{i}\right) L\left(c_{j}\right)+\sum_{i=1}^{r} \nu_{i i}(x) Q\left(c_{i}\right)
$$

with $\nu_{i j}(x) \in\left\{\left[\lambda_{i}(x), \lambda_{j}(x)\right]_{g}\right\}$. Note that $\partial g\left(\lambda_{j}(x)\right) \subseteq \mathbb{R}_{++}$is a closed convex set for every $j=1, \ldots, r$. Taking

$$
\alpha(x) \triangleq \min _{i, j}\left\{\left[\lambda_{i}(x), \lambda_{j}(x)\right]_{g}\right\}
$$

by (2.5) and the given assumptions we have $\alpha(x)>0$ and hence $\alpha(x) I \succ 0$.

We now prove $\bar{V} \triangleq V-\alpha(x) I \succeq 0$, that is, $\langle h, \bar{V} h\rangle \geq 0$ for any $h \in \mathcal{J}$. In fact, from (2.6) with $g(\lambda)=\lambda$, we have

$$
I=2 \sum_{i \neq j, i, j=1}^{r} L\left(c_{i}\right) L\left(c_{j}\right)+\sum_{i=1}^{r} Q\left(c_{i}\right) .
$$

Thus,

$$
\bar{V}=2 \sum_{i \neq j, i, j=1}^{r}\left[\nu_{i j}(x)-\alpha(x)\right] L\left(c_{i}\right) L\left(c_{j}\right)+\sum_{i=1}^{r}\left[\nu_{i i}(x)-\alpha(x)\right] Q\left(c_{i}\right)
$$

with $\left[\nu_{i j}(x)-\alpha(x)\right] \geq 0$ for any $i, j=1, \ldots, r$. Modeling the proof of Theorem 2.11, we immediately derive the desired result.

Furthermore, we can obtain the bounded property of $\partial G$ if $\partial g$ is a bounded set.

COROLlary 2.18. Under the assumptions of Theorem 2.17, for any $V \in \partial G(x)$ and scalars $a, b \in \mathbb{R}$ with $a \leq b$, there hold

(i) If $\partial g(t) \subseteq[a, b]$, then $a I \preceq V \preceq b I$.

(ii) If $\partial g(t) \subseteq(a, b)$ with $a<b$, then $a I \prec V \prec b I$.

Proof. Let $f(t)=g(t)-a t$. Note that $\partial g(t) \subseteq[a, b]$, then $\partial f(t) \subseteq \mathbb{R}_{+}$. By Theorem 2.17, one has $V-a I \succeq 0$ for any $V \in \partial G(x)$. On the other hand, letting $\bar{f}(t)=b t-g(t)$, one has $\partial \bar{f}(t) \subseteq \mathbb{R}_{+}$and hence $b I-V \succeq 0$ for any $V \in \partial G(x)$. These two arguments show part (i). Similarly, we can verify Part (ii).

3. The total NR-function. For problem (1.1), we define the natural residual function (NR-function) $\Phi_{N R}: \mathcal{J} \times \mathcal{J} \rightarrow \mathcal{J}$ by

$$
\Phi_{N R}(x, y) \triangleq x-P_{K}(x-y),
$$

and the total $N R$-function $H_{N R}: \mathcal{J} \times \mathcal{J} \rightarrow \mathcal{J} \times \mathcal{J}$ by

$$
H_{N R}(x, y) \triangleq\left(\begin{array}{c}
\Phi_{N R}(x, y) \\
F(x)-y
\end{array}\right)
$$

Copyright ( by SIAM. Unauthorized reproduction of this article is prohibited. 
Moreover, we specify function $\Psi_{N R}: \mathcal{J} \times \mathcal{J} \rightarrow \mathbb{R}$ by

$$
\Psi_{N R}(x, y) \triangleq \frac{1}{2}\left\|H_{N R}(x, y)\right\|^{2}=\frac{1}{2}\left\|\Phi_{N R}(x, y)\right\|^{2}+\frac{1}{2}\|F(x)-y\|^{2} .
$$

From Proposition 6 in [12], we know that

$$
\Phi_{N R}(x, y)=0 \Longleftrightarrow x \in K, y \in K,\langle x, y\rangle=0 .
$$

Therefore, problem (1.1) can be reformulated as a nonsmooth system of nonlinear equations: $H_{N R}(x, y)=0$. Based on this system, we may establish various solution methods, such as nonsmooth and smoothing Newton methods, see, e.g., [9, 17] for the case of NCP. In this paper, our aim is to present a globally and quadratically convergent regularized smoothing Newton method for SCCP. For this purpose, we need to investigate strong semismoothness of $H_{N R}$, nonsingularity of $\partial H_{N R}$, and level-boundedness of $\Psi_{N R}$.

First, we present a result concerning the strong semismoothness of $H_{N R}$. Since the proof is similar to that of Theorem 4.6 in [15], it is omitted.

TheOREM 3.1. Let $F: \mathcal{J} \rightarrow \mathcal{J}$ be continuously differentiable. Then the function $H_{N R}$ defined by $(3.2)$ is semismooth at any $(x, y) \in \mathcal{J} \times \mathcal{J}$. Moreover, if $\nabla F$ is locally Lipschitzian, then $H_{N R}$ is strongly semismooth at any $(x, y) \in \mathcal{J} \times \mathcal{J}$.

Next, we address Clarke generalized Jacobian $\partial H_{N R}$. Let $T \in \partial H_{N R}(x, y)$ for any $(x, y) \in \mathcal{J} \times \mathcal{J}$. Then $T$ has the following form:

$$
T=\left(\begin{array}{cc}
I-V & \nabla F(x) \\
V & -I
\end{array}\right),
$$

where $V \in \partial P_{K}(x-y)$. Since $\partial t_{+}$equals $\{1\}$ for $t>0,[0,1]$ for $t=0$, and $\{0\}$ for $t<0$, by Corollary 2.18 (i) we have $0 \preceq V \preceq I$.

The nonsingularity result on $T$ is well-known for NCP (see, e.g., [9]) or SOCCP (see, e.g., [11]). In a similar manner, we can easily show that it is still true for SCCP, which does not need a further proof. We say that $F: \mathcal{J} \rightarrow \mathcal{J}$ is monotone (strongly monotone $)$ if for all $(x, y) \in \mathcal{J} \times \mathcal{J},\langle x-y, F(x)-F(y)\rangle \geq 0(\langle x-y, F(x)-F(y)\rangle \geq$ $\varepsilon\|x-y\|^{2}$ with some $\left.\varepsilon>0\right)$.

TheOREM 3.2. Let $F: \mathcal{J} \rightarrow \mathcal{J}$ be continuously differentiable, and $T$ be given by (3.4).

(a) If $F$ is monotone and $0 \prec V \prec I$, then $T$ is invertible for any $(x, y) \in \mathcal{J} \times \mathcal{J}$.

(b) If $F$ is strongly monotone and $0 \preceq V \preceq I$, then $T$ is invertible for any $(x, y) \in \mathcal{J} \times \mathcal{J}$.

It should be noted that if $V$ is a linear and symmetric operator from $\mathcal{J}$ into itself, then the results in this theorem are still true.

We end this section by stating a well-known result on the boundedness of the level sets $\operatorname{Lev}_{\alpha}\left(\Psi_{N R}\right) \triangleq\left\{(x, y) \in \mathcal{J} \times \mathcal{J}: \Psi_{N R}(x, y) \leq \alpha\right\}$ for $\alpha \in \mathbb{R}$, which can ensure that the sequence generated by a descent method for solving $\min \Psi_{N R}(x, y)$ has at least one accumulation point. For more details, see, e.g., $[25,36]$.

TheOrem 3.3. Let $\Psi_{N R}$ be defined by (3.3). If $F(x)$ is strongly monotone and locally Lipschitzian, then the level sets $\operatorname{Lev}_{\alpha}\left(\Psi_{N R}\right)$ are bounded for all $\alpha \in \mathbb{R}$.

4. The Chen-Mangasarian smoothing function. In the previous section, we know that the total NR-function shares the strong semismoothness property because of that of the NR-function. In order to establish the desired smoothing Newton methods, we need to smoothen the NR-function and the total NR-function. This section deals with this issue.

Copyright (c) by SIAM. Unauthorized reproduction of this article is prohibited. 
In the literature on NCP, there are two well-known classes of the smoothing functions, i.e., the Chen-Mangasarian smoothing function and the smoothed FischerBurmeister function. Recently, they were successfully extended to SDCP [6, 31] and SOCCP [11]. In what follows, we first study an extension of the Chen-Mangasarian smoothing function.

Definition 4.1. Let $\boldsymbol{\digamma}: \mathcal{X} \rightarrow \mathcal{Y}$ be a nondifferentiable function. A function $\digamma_{u}: \mathcal{X} \rightarrow \mathcal{Y}$ with a parameter vector $u \in \mathbb{R}_{+}^{q}$ is called a smoothing function of $\digamma$ if it has the following properties:

(a) $\digamma_{u}$ is continuously differentiable for any $u \in \mathbb{R}_{++}^{q}$;

(b) $\lim _{u \downarrow 0} \digamma_{u}(x)=\digamma(x)$ for any $x \in \mathcal{X}$, where $u \downarrow 0$ means $u \in \mathbb{R}_{++}^{q}, u \rightarrow 0$.

We say $\digamma_{u}$ is a uniformly smooth approach function of $\digamma$ if there is a scalar $\kappa>0$ such that

$$
\left\|\digamma_{u}(x)-\digamma(x)\right\| \leq \kappa\|u\|, \quad \forall u \in \mathbb{R}_{++}^{q}, \forall x \in \mathcal{X} .
$$

Let $\varrho \in \mathbb{R}_{++}$. For NR-function $\Phi_{N R}$ as in (3.1), we define the Chen-Mangasarian smoothing function $\Phi_{\varrho}: \mathcal{J} \times \mathcal{J} \rightarrow \mathcal{J}$ as

$$
\Phi_{\varrho}(x, y)=x-\Pi_{\varrho}(x-y),
$$

where $\Pi_{\varrho}: \mathcal{J} \rightarrow \mathcal{J}$ is specified by $\Pi_{\varrho}(z) \triangleq \varrho G(z / \varrho)$ and $G \in \mathcal{C} \mathcal{M}$. Here, $\mathcal{C} \mathcal{M}$ denotes the set of Löwner operators defined by (2.3) with $g: \mathbb{R} \rightarrow \mathbb{R}_{+}$, a continuously differentiable convex function satisfying

$$
\lim _{t \rightarrow-\infty} g(t)=0, \quad \lim _{t \rightarrow \infty}(g(t)-t)=0 \text { and } 0<g^{\prime}(t)<1 \text { for all } t \in \mathbb{R} .
$$

Two known cases of function $g$ are as follows: One is the CHKS function $g(t)=$ $\left(\sqrt{t^{2}+4}+t\right) / 2$, which was proposed by Chen and Harker [1], Kanzow [18], and Smale [28], and the other is the neural network function $g(t)=\ln \left(e^{t}+1\right)$, which was used in neural networks [2]. Based on the above definitions and Theorem 2.4, we below derive formulae for $\Phi_{\varrho}$.

Proposition 4.2. Let $\Phi_{\varrho}$ be given by (4.1). Then it holds that $\Phi_{\varrho}(x, y)=$ $x-\varrho \sum_{i=1}^{r} g\left(\lambda_{i} / \varrho\right) c_{i}$ where $\lambda_{i}, c_{i}(i=1,2, \ldots, r)$ are given by $x-y=\sum_{i=1}^{r} \lambda_{i} c_{i}$. Moveover, one has

$$
\Phi_{0}(x, y) \triangleq \lim _{\varrho \downarrow 0} \Phi_{\varrho}(x, y)=x-P_{K}(x-y) .
$$

Proof. The first part is trivial. Note that $\lim _{\varrho \downarrow 0} \varrho g\left(\lambda_{i} / \varrho\right)=\left(\lambda_{i}\right)_{+}$by (4.2). This derives that $\lim _{\varrho \downarrow 0} \Phi_{\varrho}(x, y)=x-\sum_{i=1}^{r}\left(\lambda_{i}\right)_{+} c_{i}$. The second part holds by (2.4).

4.1. Uniformly smooth approximation. The following proposition claims that $\Phi_{\varrho}$ is a uniformly smooth approximation of $\Phi_{N R}$.

Proposition 4.3. Let $\Phi_{\varrho}$ be given by (4.1). Then, for any scalars $\varrho>\nu \geq 0$, we have

$$
g(0)(\varrho-\nu) e \succeq_{K} \Phi_{\nu}(x, y)-\Phi_{\varrho}(x, y) \succ_{K} 0, \quad \forall x, y \in \mathcal{J} .
$$

Proof. In order to prove the proposition, we first consider the case where $\varrho>$ $\nu>0$. By Proposition 4.2, it is easy to verify $\Phi_{\nu}(x, y)-\Phi_{\varrho}(x, y)=\sum_{i=1}^{r}\left(\varrho g\left(\lambda_{i} / \varrho\right)-\right.$ $\nu g\left(\lambda_{i} / \nu\right) c_{i}$ where $\lambda_{i}$ and $c_{i}$ are given by $x-y=\sum_{i=1}^{r} \lambda_{i} c_{i}$. Noting that for every $i=1,2, \ldots, r, 0<\varrho g\left(\lambda_{i} / \varrho\right)-\nu g\left(\lambda_{i} / \nu\right) \leq g(0)(\varrho-\nu)$ by Lemma 3.1 in [34], we have

$$
g(0)(\varrho-\nu) e=\sum_{i=1}^{r} g(0)(\varrho-\nu) c_{i} \succeq_{K} \Phi_{\nu}(x, y)-\Phi_{\varrho}(x, y) \succ_{K} 0 .
$$

Copyright @ by SIAM. Unauthorized reproduction of this article is prohibited. 
This shows that (4.3) holds in the case of $\varrho>\nu>0$, and that $-\Phi_{\nu}$ is monotone in $\nu>0$ with respect to the partial ordering $\succ_{K}$. Taking $\nu \rightarrow 0^{+}$in (4.4), one has $g(0) \varrho e \succeq_{K} \Phi_{0}(x, y)-\Phi_{\varrho}(x, y) \succ_{K} 0$. That is, (4.4) also holds for $\varrho>\nu=0$. The proof is completed.

4.2. Differentiability. Let $g: \mathbb{R} \rightarrow \mathbb{R}_{+}$be a continuously differentiable convex function satisfying (4.2). As in [34] for the setting of NCP and in [15] for the context of SOCCP, we define for any $\varrho>0$,

$$
\begin{aligned}
& \gamma_{\varrho}(t) \triangleq \varrho g(t / \varrho), \\
& \gamma_{0}(t) \triangleq \lim _{\varrho \downarrow 0} \gamma_{\varrho}(t)=\max \{0, t\}, \\
& \gamma_{0}^{+}(t) \triangleq \lim _{\varrho \downarrow 0} \gamma_{\varrho}^{\prime}(t)= \begin{cases}0 & \text { for } t<0, \\
g^{\prime}(0) & \text { for } t=0, \\
1 & \text { for } t>0 .\end{cases}
\end{aligned}
$$

Let $z=\sum_{j=1}^{r} \lambda_{j}(z) c_{j}(z)$. By $\Pi_{\varrho}(z)=\varrho G(z / \varrho)$ with $G \in \mathcal{C M}$, Theorem 2.8 leads to

$$
\nabla \Pi_{\varrho}(z)=\nabla G(z / \varrho)=2 \sum_{i \neq j, i, j=1}^{r} a_{i j} L\left(c_{i}(z)\right) L\left(c_{j}(z)\right)+\sum_{i=1}^{r} a_{i i} Q\left(c_{i}(z)\right),
$$

where for all $i, j=1,2, \ldots, r$,

$$
a_{i j}=\left[\lambda_{i}(z) / \varrho, \lambda_{j}(z) / \varrho\right]_{g}=\left\{\begin{array}{cc}
\frac{g\left(\lambda_{i}(z) / \varrho\right)-g\left(\lambda_{j}(z) / \varrho\right)}{\lambda_{i}(z) / \varrho-\lambda_{j}(z) / \varrho} & \text { if } \lambda_{i}(z) \neq \lambda_{j}(z), \\
g^{\prime}\left(\lambda_{i}(z) / \varrho\right) & \text { if } \lambda_{i}(z)=\lambda_{j}(z) .
\end{array}\right.
$$

By (4.5), we have $\gamma_{\varrho}^{\prime}(t)=g^{\prime}(t / \varrho)$. Therefore

$$
a_{i j}=\left[\lambda_{i}(z), \lambda_{j}(z)\right]_{\gamma_{\varrho}}=\left\{\begin{array}{cc}
\frac{\gamma_{\varrho}\left(\lambda_{i}(z)\right)-\gamma_{\varrho}\left(\lambda_{j}(z)\right)}{\lambda_{i}(z)-\lambda_{j}(z)} & \text { if } \lambda_{i}(z) \neq \lambda_{j}(z), \\
\gamma_{\varrho}^{\prime}\left(\lambda_{i}(z)\right) & \text { if } \lambda_{i}(z)=\lambda_{j}(z) .
\end{array}\right.
$$

By (4.2) and (4.9), one has $0<a_{i j}<1$. Thus, by Corollary 2.18 (ii), it holds $I \succ \nabla \Pi_{\varrho}(z) \succ 0$. In summary, we have the following conclusion.

Proposition 4.4. The function $\Pi_{\varrho}$ is continuously differentiable, and $I \succ$ $\nabla \Pi_{\varrho}(z) \succ 0$.

Furthermore, by applying Theorem 2.8 and the chain rule, we immediately obtain the differential property of the Chen-Mangasarian smoothing function $\Phi_{\varrho}$, which does not need a proof.

Proposition 4.5. For any $\varrho>0$, the Chen-Mangasarian smoothing function $\Phi_{\varrho}$, defined by (4.1), is continuously differentiable and its Jacobian is given by

$$
\nabla \Phi_{\varrho}(x, y)=\left(\begin{array}{c}
I-\nabla \Pi_{\varrho}(x-y) \\
\nabla \Pi_{\varrho}(x-y)
\end{array}\right)=\left(\begin{array}{c}
I-\nabla G((x-y) / \varrho) \\
\nabla G((x-y) / \varrho)
\end{array}\right) .
$$

4.3. Jacobian consistency. Like strong semismoothness, Jacobian consistency plays an important role in establishing rapid convergence of smoothing Newton methods. This concept was originally introduced by Chen, Qi, and Sun [8] for variational 
inequalities, and was recently used by Hayashi, Yamashita, and Fukushima [15] analyzing the regularized smoothing method for SOCCP, where their Jacobian consistency contains two parameters. We state a more general definition as follows.

Definition 4.6. Suppose that $\digamma: \mathcal{X} \rightarrow \mathcal{Y}$ is a continuous function and $\partial \digamma$ exists. Let $\digamma_{u}$ be a smoothing function of $\digamma$. We say that $\digamma_{u}$ satisfies the Jacobian consistency if

$$
\lim _{u \downarrow 0} \operatorname{dist}\left(\nabla \digamma_{u}(x), \partial \digamma(x)\right)=0, \text { for any } x \in \mathcal{X} .
$$

To show Jacobian consistency of the Chen-Mangasarian smoothing function $\Phi_{\varrho}$, we first look at the function $\Pi_{\varrho}(z)$. Define $b_{i j} \triangleq \lim _{\varrho \downarrow 0} a_{i j}$ for all $i, j=1,2, \ldots, r$. From (4.5)-(4.7) and (4.9), we derive that

$$
b_{i j}=\left\{\begin{array}{cc}
\frac{\gamma_{0}\left(\lambda_{i}(z)\right)-\gamma_{0}\left(\lambda_{j}(z)\right)}{\lambda_{i}(z)-\lambda_{j}(z)} & \text { if } \lambda_{i}(z) \neq \lambda_{j}(z) \\
\gamma_{0}^{+}\left(\lambda_{i}(z)\right) & \text { if } \lambda_{i}(z)=\lambda_{j}(z) .
\end{array}\right.
$$

Obviously, by (4.2), $0 \leq b_{i j} \leq 1$. By the direct calculation, one has

$$
\lim _{\varrho \downarrow 0} \nabla \Pi_{\varrho}(z)=2 \sum_{i \neq j, i, j=1}^{r} b_{i j} L\left(c_{i}(z)\right) L\left(c_{j}(z)\right)+\sum_{i=1}^{r} b_{i i} Q\left(c_{i}(z)\right) .
$$

Rewriting $z$ as $z=\sum_{i=1}^{\bar{r}} \mu_{i}(z) b_{i}(z)$, from Theorem 2.8 we deduce

$$
\nabla \Pi_{\varrho}(z)=2 \sum_{i \neq j, i, j=1}^{\bar{r}}\left[\mu_{i}(z), \mu_{j}(z)\right]_{\gamma_{\varrho}} L\left(b_{i}(z)\right) L\left(b_{j}(z)\right)+\sum_{i=1}^{\bar{r}} \gamma_{\varrho}^{\prime}\left(\mu_{i}(z)\right) Q\left(b_{i}(z)\right) .
$$

In a similar manner as in (4.12), we derive that

$$
\lim _{\varrho \downarrow 0} \nabla \Pi_{\mu}(z)=2 \sum_{i \neq j, i, j=1}^{\bar{r}}\left[\mu_{i}(z), \mu_{j}(z)\right]_{\gamma_{0}} L\left(b_{i}(z)\right) L\left(b_{j}(z)\right)+\sum_{i=1}^{\bar{r}} \gamma_{0}^{+}\left(\mu_{i}(z)\right) Q\left(b_{i}(z)\right) .
$$

Take $\partial_{\Pi}^{0}(z) \triangleq \lim _{\varrho \downarrow 0} \nabla \Pi_{\mu}(z)$. It follows from Theorem 2.14 that $\partial_{\Pi}^{0}(z) \in \underline{\partial} P_{K}(z) \subseteq$ $\partial P_{K}(z)$. Summarizing the preceding argument, we have the following.

Lemma 4.7. Let $\partial_{\Pi}^{0}(z)=\lim _{\varrho \downarrow 0} \nabla \Pi_{\varrho}(z)$. Then $\partial_{\Pi}^{0}(z) \in \partial P_{K}(z)$ for any $z \in \mathcal{J}$. Thus $\Pi_{\varrho}$ satisfies the Jacobian consistency.

Combining Lemma 4.7 with Proposition 4.5, the Jacobian consistency of $\Phi_{\varrho}$ is immediate.

Proposition 4.8. $\Phi_{\varrho}$ satisfies the Jacobian consistency.

In the end of this section, we further consider the function $g$ satisfying both (4.2) and the following

$$
g(t)-t=g(-t), \quad \forall t \in \mathbb{R} .
$$

For instance, $\left(\sqrt{t^{2}+4}+t\right) / 2$ and $\ln \left(e^{t}+1\right)$ are such two functions. Can we get a more specific result than Proposition 4.8 in this case? To settle this question, we need the following lemma from [15].

Lemma 4.9 (Lemma 4.10, [15]). Let $g$ be a continuously differentiable convex function satisfying (4.2) and (4.13). Let $\gamma_{\varrho}, \gamma_{0}$, and $\gamma_{0}^{+}$be given by (4.5)-(4.7). Then it holds that 
(a) $\gamma_{\varrho}(t)-\gamma_{0}(t)=\gamma_{\varrho}(-t)-\gamma_{0}(-t)$ for any $t \in \mathbb{R}$;

(b) $\left|\gamma_{\varrho}^{\prime}(t)-\gamma_{0}^{+}(t)\right|=\left|\gamma_{\varrho}^{\prime}(|t|)-\gamma_{0}^{+}(|t|)\right|$ for any $t \in \mathbb{R}$;

(c) $\left|\gamma_{\varrho}^{\prime}(0)-\gamma_{0}^{+}(0)\right|=0<\left|\gamma_{\varrho}^{\prime}\left(t_{2}\right)-\gamma_{0}^{+}\left(t_{2}\right)\right| \leq\left|\gamma_{\varrho}^{\prime}\left(t_{1}\right)-\gamma_{0}^{+}\left(t_{1}\right)\right|$ for any $t_{i} \in$ $\mathbb{R}(i=1,2)$ such that $0<\left|t_{1}\right| \leq\left|t_{2}\right|$.

For $z=\sum_{j=1}^{r} \lambda_{j}(z) c_{j}(z)$, let $N(z)$ be the index set specified by $N(z) \triangleq\{i$ : $\left.\lambda_{i}(z) \neq 0\right\}$. Define the function $\tilde{\lambda}: \mathcal{J} \rightarrow \mathbb{R}_{+}$by

$$
\tilde{\lambda}(z) \triangleq\left\{\begin{array}{cc}
\min _{i \in N(z)}\left|\lambda_{i}(z)\right| & \text { for } N(z) \neq \varnothing \\
0 & \text { for } N(z)=\varnothing
\end{array}\right.
$$

Obviously, $\tilde{\lambda}(z)=0$ if and only if $z=0$. When $z \neq 0$, by (4.5) and the continuous differentiability of $g$, there is a scalar $\varsigma \in(0, \tilde{\lambda}(z))$ such that $\gamma_{\varrho}^{\prime}(\varsigma)=\frac{\gamma_{\varrho}(\tilde{\lambda}(z))-\gamma_{\varrho}(0)}{\tilde{\lambda}(z)}$; meanwhile, noting that $g$ is convex, one has $\gamma_{\varrho}^{\prime}(t) \leq \frac{\gamma_{\varrho}(\tilde{\lambda}(z))-\gamma_{\varrho}(0)}{\tilde{\lambda}(z)}$ for any $t \in(0, \varsigma)$. So, in the case of $z \neq 0$, there exists a positive integer $l$ such that $\frac{1}{2^{2}} \tilde{\lambda}(z) \in(0, \varsigma)$.

Based on the preceding argument, we define the function $\lambda^{\star}: \mathcal{J} \rightarrow \mathbb{R}_{+}$by

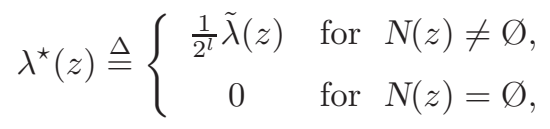

where $l$ is the smallest positive integer such that

$$
\gamma_{\varrho}^{\prime}\left(\frac{1}{2^{l}} \tilde{\lambda}(z)\right) \leq \frac{\gamma_{\varrho}(\tilde{\lambda}(z))-\gamma_{\varrho}(0)}{\tilde{\lambda}(z)} .
$$

Then $\lambda^{\star}(z)$ is well-defined and $0<\lambda^{\star}(z)<\tilde{\lambda}(z)$. Thus, it holds by Lemma 4.9 (c) that

$$
\begin{aligned}
\left|\gamma_{\varrho}^{\prime}\left(\lambda_{i}(z)\right)-\gamma_{0}^{+}\left(\lambda_{i}(z)\right)\right| & \leq\left|\gamma_{\varrho}^{\prime}(\tilde{\lambda}(z))-\gamma_{0}^{+}(\tilde{\lambda}(z))\right| \\
& \leq\left|\gamma_{\varrho}^{\prime}\left(\lambda^{\star}(z)\right)-\gamma_{0}^{+}\left(\lambda^{\star}(z)\right)\right|, i=1,2, \ldots, r .
\end{aligned}
$$

Now we are ready to claim that $\Pi_{\varrho}(z)$ not only satisfies the Jacobian consistency but also has the stronger Jacobian property.

THEOREM 4.10. Let $\partial_{\Pi}^{0}(z)=\lim _{\varrho \downarrow 0} \nabla \Pi_{\varrho}(z)$. Suppose $g$ is a continuously differentiable convex function satisfying (4.2) and (4.13). Let $\gamma_{\varrho}, \gamma_{0}, \gamma_{0}^{+}$, and $\lambda^{\star}$ be given by (4.5)-(4.7) and (4.15), respectively. Then there exists a scalar $\bar{M}>0$ such that

$$
\left\|\nabla \Pi_{\varrho}(z)-\partial_{\Pi}^{0}(z)\right\| \leq \bar{M}\left|\gamma_{\varrho}^{\prime}\left(\lambda^{\star}(z)\right)-\gamma_{0}^{+}\left(\lambda^{\star}(z)\right)\right|, \quad \forall \varrho \in \mathbb{R}_{++}, \quad \forall z \in \mathcal{J} .
$$

Proof. Let $z=\sum_{j=1}^{r} \lambda_{j}(z) c_{j}(z)$. Then from (4.8) and (4.12) we obtain

$$
\nabla \Pi_{\varrho}(z)-\partial_{\Pi}^{0}(z)=2 \sum_{i \neq j, i, j=1}^{r}\left(a_{i j}-b_{i j}\right) L\left(c_{i}(z)\right) L\left(c_{j}(z)\right)+\sum_{i=1}^{r}\left(a_{i i}-b_{i i}\right) Q\left(c_{i}(z)\right) .
$$

To prove the theorem, it is enough to show $\left|a_{i j}-b_{i j}\right| \leq\left|\gamma_{\varrho}^{\prime}\left(\lambda^{\star}(z)\right)-\gamma_{0}^{+}\left(\lambda^{\star}(z)\right)\right|$ for every $i, j=1,2, \ldots, r$. We consider below two cases. 
Case (i): $0=\lambda_{i}(z)<\left|\lambda_{j}(z)\right|$. By (4.9) and (4.11), the direct calculation yields

$$
\begin{aligned}
\left|a_{i j}-b_{i j}\right| & =\left|\frac{\gamma_{\varrho}(0)-\gamma_{\varrho}\left(\lambda_{j}(z)\right)}{0-\lambda_{j}(z)}-\frac{\gamma_{0}(0)-\gamma_{0}\left(\lambda_{j}(z)\right)}{0-\lambda_{j}(z)}\right| \\
& =\left|\frac{\gamma_{\varrho}\left(\lambda_{j}(z)\right)-\gamma_{\varrho}(0)}{\lambda_{j}(z)}-1\right| \\
& =1-\frac{\gamma_{\varrho}\left(\lambda_{j}(z)\right)-\gamma_{\varrho}(0)}{\lambda_{j}(z)} \\
& \leq 1-\gamma_{\varrho}^{\prime}\left(\lambda^{\star}(z)\right) \\
& =\left|\gamma_{\varrho}^{\prime}\left(\lambda^{\star}(z)\right)-\gamma_{0}^{+}\left(\lambda^{\star}(z)\right)\right|,
\end{aligned}
$$

where the second equality follows from the fact $\frac{\gamma_{0}(0)-\gamma_{0}\left(\lambda_{j}(z)\right)}{0-\lambda_{j}(z)}=1$ by $(4.6)$, the third one from $0<\frac{\gamma_{\varrho}\left(\lambda_{j}(z)\right)-\gamma_{\rho}(0)}{\lambda_{j}(z)}=\frac{g\left(\lambda_{j}(z) / \varrho\right)-g(0)}{\lambda_{j}(z) / \varrho}<1$ by $(4.2)$, the inequality from (4.15), and the last equality from $\gamma_{0}^{+}\left(\lambda^{\star}(z)\right)=1$ by (4.15) and (4.7).

Case (ii): Otherwise, one has $\left|a_{i j}-b_{i j}\right| \leq\left|\gamma_{\varrho}^{\prime}(\tilde{\lambda}(z))-\gamma_{0}^{+}(\tilde{\lambda}(z))\right|$, whose proof is perfectly similar to that in [15] and is omitted for brevity.

5. Regularized smoothing function and algorithm. Based on the proceeding results, we shall develop the Chen-Mangasarian class of regularized smoothing functions for SCCP, and derive the regularized smoothing Newton method for solving the monotone SCCP.

For the given $F$ in (1.1) and a parameter $\varepsilon>0$, we define a new function $F_{\varepsilon}$ : $\mathcal{J} \rightarrow \mathcal{J}$ as

$$
F_{\varepsilon}(x) \triangleq F(x)+\varepsilon x .
$$

Again, define functions $H_{\varrho, \varepsilon}: \mathcal{J} \times \mathcal{J} \rightarrow \mathcal{J} \times \mathcal{J}$ and $\Psi_{\varrho, \varepsilon}: \mathcal{J} \times \mathcal{J} \rightarrow \mathbb{R}$ by

$$
\begin{aligned}
& H_{\varrho, \varepsilon}(x, y) \triangleq\left(\begin{array}{c}
\Phi_{\varrho}(x, y) \\
F_{\varepsilon}(x)-y
\end{array}\right), \\
& \Psi_{\varrho, \varepsilon}(x, y) \triangleq \frac{1}{2}\left\|H_{\varrho, \varepsilon}(x, y)\right\|^{2}=\frac{1}{2}\left\|\Phi_{\varrho}(x, y)\right\|^{2}+\frac{1}{2}\left\|F_{\varepsilon}(x)-y\right\|^{2} .
\end{aligned}
$$

Then, $H_{\varrho, \varepsilon}$ is a smoothing approximation of the regularized SCCP involving $F_{\varepsilon}$ with $\varepsilon>0$. Obviously, if $F$ is monotone, then $F_{\varepsilon}$ is strongly monotone for any $\varepsilon>0$. In addition, if $F$ is also locally Lipschitzian, then $\Psi_{\varrho, \varepsilon}$ is level-bounded for any $\varrho \geq 0$ and $\varepsilon>0$ via Theorem 3.3.

The proposed method applies the Newton algorithm to the system $H_{\varrho, \varepsilon}(x, y)=0$ with $\varrho$ and $\varepsilon$ properly adjusted at each iteration, so that a solution of the original SCCP is eventually obtained by taking the limits as $\varrho \downarrow 0$ and $\varepsilon \downarrow 0$.

For this purpose, we deal with $H_{\varrho, \varepsilon}$. From Proposition 4.5, we obtain

$$
\nabla H_{\varrho, \varepsilon}(x, y)=\left(\begin{array}{cc}
I-\nabla \Pi_{\varrho}(x-y) & \nabla F(x)+\varepsilon I \\
\nabla \Pi_{\varrho}(x-y) & -I
\end{array}\right),
$$

where $\nabla \Pi_{\varrho}(\cdot)$ is specified by $(4.8)$.

From (5.4) and Proposition 4.4, one can easily get the nonsingularity of $\nabla H_{\varrho, \varepsilon}$. The proof is omitted.

TheOREM 5.1. Let $F: \mathcal{J} \rightarrow \mathcal{J}$ be continuously differentiable. For parameters $\varrho>0$ and $\varepsilon>0$, let $\Phi_{\varrho}(x, y), F_{\varepsilon}(x)$, and $H_{\varrho, \varepsilon}(x, y)$ be defined by (4.1), (5.1), and 
(5.2), respectively. If $F$ is monotone, then $\nabla H_{\varrho, \varepsilon}$, given by (5.4), is invertible for any $(x, y) \in \mathcal{J} \times \mathcal{J}$.

In view of (5.4), we also deduce the Jacobian consistency of $H_{\varrho, \varepsilon}$.

TheOREM 5.2. Let $F: \mathcal{J} \rightarrow \mathcal{J}$ be continuously differentiable. For parameters $\varrho>0$ and $\varepsilon>0$, let $\Phi_{\varrho}(x, y), F_{\varepsilon}(x)$, and $H_{\varrho, \varepsilon}(x, y)$ be defined by (4.1), (5.1), and (5.2), respectively. Then $H_{\varrho, \varepsilon}$ satisfies the Jacobian consistency.

Proof. It holds by (5.4) and $\partial_{\Pi}^{0}(z)=\lim _{\varrho \downarrow 0} \nabla \Pi_{\varrho}(z)$ that

$$
\partial_{\Pi}^{0} H(x, y) \triangleq \lim _{(\varrho, \varepsilon) \downarrow(0,0)} \nabla H_{\varrho, \varepsilon}(x, y)=\left(\begin{array}{cc}
I-\partial_{\Pi}^{0}(x-y) & \nabla F(x) \\
\partial_{\Pi}^{0}(x-y) & -I
\end{array}\right) .
$$

This implies from (3.4) and Lemma 4.7 that $\partial_{\Pi}^{0} H(x, y) \in \partial H_{N R}(x, y)$ for any $(x, y) \in$ $\mathcal{J} \times \mathcal{J}$. The desired conclusion holds obviously.

Furthermore, applying Theorems 4.10 and 5.2, we estimate the upper bound of the distance $\operatorname{dist}\left(\nabla H_{\varrho, \varepsilon}(x, y), \partial H_{N R}(x, y)\right)$.

ThEOREM 5.3. Let $F: \mathcal{J} \rightarrow \mathcal{J}$ be continuously differentiable, and $g$ be a continuously differentiable convex function satisfying (4.2) and (4.13). Suppose $\gamma_{\varrho}, \gamma_{0}$, and $\gamma_{0}^{+}$are given by (4.5)-(4.7), and let $\lambda^{\star}$ be defined by (4.15). Then, there exists a scalar $M>0$ such that

$$
\operatorname{dist}\left(\nabla H_{\varrho, \varepsilon}(x, y), \partial H_{N R}(x, y)\right) \leq M\left(\left|\gamma_{\varrho}^{\prime}\left(\lambda^{\star}(x-y)\right)-\gamma_{0}^{+}\left(\lambda^{\star}(x-y)\right)\right|+\varepsilon\right),
$$

for any $\varrho>0, \varepsilon \geq 0$ and any $(x, y) \in \mathcal{J} \times \mathcal{J}$.

Proof. By (5.4), (5.5), and the fact $\partial_{\Pi}^{0} H(x, y) \in \partial H_{N R}(x, y)$, one has for any $\varrho>0, \varepsilon \geq 0$, and any $(x, y) \in \mathcal{J} \times \mathcal{J}$,

$$
\begin{aligned}
\operatorname{dist}\left(\nabla H_{\varrho, \varepsilon}(x, y), \partial H_{N R}(x, y)\right) & \leq\left\|\nabla H_{\varrho, \varepsilon}(x, y)-\partial_{\Pi}^{0} H(x, y)\right\| \\
& \left.\left.\leq \tilde{M}\left(\| \nabla \Pi_{\varrho}(x-y)\right)-\partial_{\Pi}^{0}(x-y)\right) \|+\varepsilon\right) \\
& \leq \tilde{M}\left(\bar{M}\left|\gamma_{\varrho}^{\prime}\left(\lambda^{\star}(x-y)\right)-\gamma_{0}^{+}\left(\lambda^{\star}(x-y)\right)\right|+\varepsilon\right)
\end{aligned}
$$

where $\tilde{M}$ in the second inequality is a positive scalar, the third follows from Theorem 4.10. The desired holds immediately.

In the end of this paper, we describe an algorithm which is a word-for-word extension of the one by Hayashi, Yamashita, and Fukushima [15] for SOCCP, and state the corresponding convergence theorem, which can be obtained by Theorems 5.1-5.3 and following the proof of Theorem 4.13 in [15].

ALGORITHM Set $w \triangleq(x, y)$ and $w^{(k)} \triangleq\left(x^{(k)}, y^{(k)}\right)$. Choose $\eta, \rho \in(0,1), \bar{\eta} \in$ $(0, \eta], \sigma \in(0,1 / 2), \kappa>0$, and $\hat{\kappa}>0$.

Step 0 Choose $w^{(0)} \in \mathcal{J} \times \mathcal{J}$ and $\beta_{0} \in(0, \infty)$. Let $\varrho_{0} \triangleq\left\|H_{N R}\left(w^{(0)}\right)\right\|$ and $\varepsilon_{0} \triangleq\left\|H_{N R}\left(w^{(0)}\right)\right\|$. Set $k \triangleq 0$.

Step 1 Terminate if $\left\|H_{N R}\left(w^{(k)}\right)\right\|=0$.

Step 2

Step 2.0 Set $v^{(0)} \triangleq w^{(0)}$ and $j \triangleq 0$.

Step 2.1 Find a vector $\hat{d}^{(j)}$ such that

$$
H_{\varrho_{k}, \varepsilon_{k}}\left(v^{(j)}\right)+\nabla H_{\varrho_{k}, \varepsilon_{k}}\left(v^{(j)}\right)^{T} \hat{d}^{(j)}=0
$$

Step 2.2 If $\left\|H_{\varrho_{k}, \varepsilon_{k}}\left(v^{(j)}+\hat{d}^{(j)}\right)\right\| \leq \beta_{k}$, then let $w^{(k+1)} \triangleq v^{(j)}+\hat{d}^{(j)}$ and go to Step 3. Otherwise, go to Step 2.3.

Copyright (C) by SIAM. Unauthorized reproduction of this article is prohibited. 
Step 2.3 Find the smallest nonnegative integer $m$ such that

$$
\Psi_{\varrho_{k}, \varepsilon_{k}}\left(v^{(j)}+\rho^{m} \hat{d}^{(j)}\right) \leq\left(1-2 \sigma \rho^{m}\right) \Psi_{\varrho_{k}, \varepsilon_{k}}\left(v^{(j)}\right) .
$$

Let $m_{j} \triangleq m, \tau_{j} \triangleq \rho^{m_{j}}$, and $v^{(j+1)} \triangleq v^{(j)}+\tau_{j} \hat{d}^{(j)}$.

Step 2.4 If $\left\|H_{\varrho_{k}, \varepsilon_{k}}\left(v^{(j+1)}\right)\right\| \leq \beta_{k}$, then let $w^{(k+1)} \triangleq v^{(j+1)}$ and go to

Step 3. Otherwise, set $j \triangleq j+1$ and go back to Step 2.1.

Step 3 Update the parameters as follows:

$$
\begin{aligned}
\varrho_{k+1}:= & \min \left\{\kappa\left\|H_{N R}\left(w^{(k+1)}\right)\right\|^{2}, \varrho_{0} \bar{\eta}^{k+1}, \varrho\left(\lambda^{\star}\left(x^{(k+1)}-y^{(k+1)}\right), \hat{\kappa}\right.\right. \\
& \left.\left.\left\|H_{N R}\left(w^{(k+1)}\right)\right\|\right)\right\}, \\
\varepsilon_{k+1}:= & \min \left\{\kappa\left\|H_{N R}\left(w^{(k+1)}\right)\right\|^{2}, \varepsilon_{0} \bar{\eta}^{k+1}\right\}, \\
\beta_{k+1}:= & \beta_{0} \eta^{k+1},
\end{aligned}
$$

where $\lambda^{\star}$ is given by $(4.15)$, and $\bar{\varrho}(t, \delta)$ is determined so that $\mid \gamma_{\varrho}^{\prime}(t)-$ $\gamma_{0}^{+}(t) \mid<\delta$ for any $\varrho \in(0, \bar{\varrho}(t, \delta))$.

Step 4 Set $k \triangleq k+1$. Go back to Step 1 .

Note that by (4.14)-(4.16) it is not hard to calculate $\lambda^{\star}$ for NCP, SOCCP, and SDCP cases.

THEOREM 5.4. Let $F: \mathcal{J} \rightarrow \mathcal{J}$ be a continuously differentiable and monotone function, and $\left\{w^{(k)}\right\}$ be a sequence generated by the Algorithm. If the solution set of $S C C P(1.1)$ is nonempty and bounded, then $\left\{w^{(k)}\right\}$ is bounded, and every accumulation point is a solution of $S C C P(1.1)$. In addition, if $\nabla F$ is locally Lipschitzian and every accumulation point of $\left\{\nabla H_{\varrho_{k}, \varepsilon_{k}}\left(w^{(k)}\right)\right\}$ is nonsingular, then the sequence $\left\{w^{(k)}\right\}$ converges to a solution $w^{*}$ of $S C C P(1.1)$ quadratically.

Acknowledgments. The authors are very grateful to the anonymous referees for their constructive comments and valuable suggestions. The authors thank Defeng Sun and Levent Tunçel for helpful discussions.

\section{REFERENCES}

[1] B. Chen And P. T. Harker, A non-interior-point continuation method for linear complementarity problems, SIAM J. Matrix Anal. Appl., 14 (1993), pp. 1168-1190.

[2] C. Chen and O. L. Mangasarian, Smoothing methods for convex inequalities and linear complementarity problems, Math. Program., 71 (1995), pp. 51-69.

[3] J.-S. Chen And P. TSEng, An unconstrained smooth minimization reformulation of the secondorder cone complementarity problem, Math. Program. Ser. B, 104 (2005), pp. 293-327.

[4] X. Chen And H. D. QI, Cartesian P-property and its applications to the semidefinite linear complementarity problem, Math. Program., 106 (2006), pp. 177-201.

[5] X. Chen, H. D. QI, AND P. Tseng, Analysis of nonsmooth symmetric matrix functions with applications to semidefinite complementarity problems, SIAM J. Optim., 13 (2003), pp. 960985.

[6] X. Chen and P. Tseng, Non-interior continuation methods for solving semidefinite complementarity problems, Math. Program., 95 (2003), pp. 431-474.

[7] X. D. Chen, D. Sun, And J. Sun, Complementarity functions and numerical experiments on some smoothing Newton methods for second-order-cone complementarity problems, Comput. Optim. Appl., 25 (2003), pp. 39-56.

[8] X. J. Chen, L. QI, AND D. Sun, Global and superlinear convergence of the smoothing Newton method and its application to general box constrained variational inequalities, Math. Comput., 67 (1998), pp. 519-540. 
[9] F. Facchinei And J.-S. PAng, Finite-Dimensional Variational Inequalities and Complementarity Problems, Volume I and II, Springer-Verlag, New York, 2003.

[10] J. Faraut and A. Korányi, Analysis on Symmetric Cones, Oxford University Press, New York, 1994.

[11] M. Fukushima, Z. Q. Luo, and P. Tseng, Smoothing functions for second-order cone complementarity problems, SIAM J. Optim., 12 (2001), pp. 436-460.

[12] M. S. Gowda, R. Sznajder, And J. TaO, Some P-properties for linear transformations on Euclidean Jordan algebras, Linear Algebra Appl., 393 (2004), pp. 203-232.

[13] M. S. Gowda And R. Sznajder, Automorphism invariance of $P$ and GUS properties of linear transformations on Euclidean Jordan algebras, Math. Oper. Res., 31 (2006), pp. 109-123.

[14] M. S. Gowda AND R. SznAJder, Some global uniqueness and solvability results for linear complementarity problems over symmetric cones, SIAM J. Optim., 18 (2007), pp. 461481.

[15] S. Hayashi, N. Yamashita, and M. Fukushima, A combined smoothing and regularization method for monotone second-order cone complementarity problems, SIAM J. Optim., 15 (2005), pp. 593-615.

[16] Z. H. HuANG AND J. HAN, Non-interior continuation method for solving the monotone semidefinite complementarity problem, Appl. Math. Optim., 47 (2003), pp. 195-211.

[17] G. IsAC, Topological Methods in Complementarity Theory, Kluwer Academic Publishers, Dordrecht, 2000

[18] C. Kanzow, Some noninterior continuation methods for linear complementarity problems, SIAM J. Matrix Anal. Appl., 17 (1996), pp. 851-868.

[19] M. Koecher, The Minnesota Notes on Jordan Algebras and Their Applications, edited and annotated by A. Brieg and S. Walcher, Springer, Berlin, 1999.

[20] L. C. Kong And N. H. XIU, On uniqueness of the Jordan frame in Euclidean Jordan algebras, J. Beijing Jiaotong University, 3 (2007), pp. 54-57.

[21] K. LÖWNER, Über monotone matrixfunctionen, Math. Z., 38 (1934), pp. 177-216.

[22] Y. Liu, L. Zhang, And Y. Wang, Some properties of a class of merit functions for symmetric cone complementarity problems, Asia-Pacific J. Oper. Res., 23 (2006), pp. 473-496.

[23] M. Malik and S. R. Mohan, Cone complementarity problems with finite solution sets, Oper. Res. Lett., 34 (2006), pp. 121-126.

[24] R. Mifflin, Semismooth and semiconvex functions in constrained optimization, SIAM J. Cont. Optim., 15 (1977), pp. 959-972.

[25] J. M. Peng And M. Fukushima, A hybrid Newton method for solving the variational inequality problem via the D-gap function, Math. Program., 86 (1999), pp. 367-386.

[26] L. Qi And J. Sun, A nonsmooth version of Newton's method, Math. Program., 58 (1993), pp. 353-367.

[27] R. T. Rockafellar and R. J.-B. Wets, Variational Analysis, Springer, Berlin, 2004.

[28] S. Smale, Algorithms for solving equations, in Proceedings of the International Congress of Mathematicians, American Mathematical Society, Providence, RI, 1987, pp. 172-195.

[29] D. Sun And J. Sun, Semismooth matrix valued functions, Math. Oper. Res., 27 (2002), pp. 150169.

[30] D. Sun AND J. Sun, Löwner's operator and spectral functions on Euclidean Jordan algebras, Math. Oper. Res., 33 (2008), pp. 421-445.

[31] D. Sun And J. Sun, Strong semismoothness of the Fischer-Burmeister SDC and SOC complementarity functions, Math. Program., 103 (2005), pp. 575-582.

[32] J. Sun, D. Sun, AND L. QI, A squared smoothing Newton method for nonsmooth matrix equations and its applications in semidefinite optimization problems, SIAM J. Optim., 14 (2004), pp. 783-806.

[33] J. TAO And M. S. Gowda, Some P-properties for nonlinear transformations on Euclidean Jordan algebras, Math. Oper. Res., 30 (2005), pp. 985-1004.

[34] P. Tseng, Analysis of a non-interior continuation method based on Chen-Mangasarian smoothing functions for complementarity problems, in Reformulation-Nonsmooth, Piecewise Smooth, Semismooth and Smoothing Methods, M. Fukushima, L. Qi, eds., Kluwer Academic, Boston, 1999, pp. 381-404.

[35] Y. XIA AND J. M. Peng, A continuation method for the linear second-order cone complementarity Problem, Computational Science and Its Applications-ICCSA 2005, Vol. 4, Proceedings Lecture Notes in Computer Science 3483, Springer, Berlin, 2005, pp. 290-300.

[36] N. Yamashita And M. Fukushima, On the level-boundedness of the natural residual function for variational inequality problems, Pac. J. Optim., 1 (2005), pp. 625-630.

[37] A. Yoshise, Interior point trajectories and a homogeneous model for nonlinear complementarity problems over symmetric cones, SIAM J. Optim., 17 (2006), pp. 1129-1153.

Copyright (c) by SIAM. Unauthorized reproduction of this article is prohibited. 